ACCEPted to ApJ

Preprint typeset using $\mathrm{IATEX}_{\mathrm{E}}$ style emulateapj v. 08/22/09

\title{
MASS MODELS FOR LOW SURFACE BRIGHTNESS GALAXIES WITH HIGH RESOLUTION OPTICAL VELOCITY FIELDS
}

\author{
RACHEL KUZIO DE NARAY ${ }^{1,2}$ \\ Center for Cosmology, Department of Physics and Astronomy, University of California, Irvine, CA 92697-4575
}

\author{
Stacy S. MCGaugh \\ Department of Astronomy, University of Maryland, College Park, MD 20742-2421 \\ AND \\ W. J. G. DE BLOK \\ Department of Astronomy, University of Cape Town, Rondebosch 7700, South Africa \\ Accepted to ApJ
}

\begin{abstract}
We present high-resolution optical velocity fields from DensePak integral field spectroscopy, along with derived rotation curves, for a sample of low surface brightness galaxies. In the limit of no baryons, we fit the NFW and pseudoisothermal halo models to the data and find the rotation curve shapes and halo central densities to be better described by the isothermal halo. For those galaxies with photometry, we present halo fits for three assumptions of the stellar mass-to-light ratio, $\Upsilon_{*}$. We find that the velocity contribution from the baryons is significant enough in the maximum disk case that maximum disk and the NFW halo are mutually exclusive. We find a substantial cusp mass excess at the centers of the galaxies, with at least two times more mass expected in the cuspy CDM halo than is allowed by the data. We also find that to reconcile the data with $\Lambda \mathrm{CDM}, \sim 20 \mathrm{~km} \mathrm{~s}^{-1}$ noncircular motions are needed and/or the power spectrum has a lower amplitude on the scales we probe.

Subject headings: dark matter — galaxies: fundamental parameters - galaxies: kinematics and dynamics
\end{abstract}

\section{INTRODUCTION}

The behavior of cold dark matter (CDM) on galaxy scales has been discussed extensively in the literature, with particular attention given to constraints from rotation curves of low surface brightness (LSB) galaxies. Numerical simulations of CDM predict cuspy halos with a density distribution showing a $\rho \sim r^{-\alpha}(\alpha \gtrsim 1)$ behavior, regardless of mass (e.g., Cole \& Lacev 1996; Navarro, Frenk, \& White 1996, 1997; Avila-Reese, Firmani, \& Hernandez 1998; Klypin et al. 2001; Diemand et al. 2005). However, numerous studies of the rotation curves of LSB and dwarf galaxies have found the data to be inconsistent with a cuspy halo, and instead to be more consistent with a halo having a nearly constant density core: $\rho \sim r^{-\alpha}(\alpha \approx 0)$ (e.g., Flores \& Primack 1994; de Blok, McGaugh, \& van der Hulst 1996; McGaugh. Rubin, \& de Blok 2001; Marchesini et al. 2002; de Blok. Bosma, \& McGaugh 2003; Gentile et al. 2004, 2005; Simon et al. 2005; Kuzio de Naray et al. 2006; Spano et al. 2007). This interpretation of the observations has been met with skepticism, though, as cored halos lack theoretical and cosmological moti-

Electronic address: kuzio@uci.edu

Electronic address: ssm@astro.umd.edu

Electronic address: edeblok@circinus.ast.uct.ac.za

${ }^{1}$ NSF Astronomy and Astrophysics Postdoctoral Fellow

${ }^{2}$ Visiting Astronomer, Kitt Peak National Observatory, National Optical Astronomy Observatory, which is operated by the Association of Universities for Research in Astronomy, Inc. (AURA) under cooperative agreement with the National Science Foundation. vation. Systematic effects in the data (most notably beam smearing, slit misplacement, and noncircular motions) have also been used to argue against the presence of cored halos (e.g., van den Bosch et al.|2000; Swaters et al. 2003a; Simon et al. 2003; Rhee et al. 2004), although some authors find the magnitude of these effects to be insufficient for masking the presence of a cusp (de Blok et al. 2003).

To address these concerns, the most recent observations have been made using integral field spectrographs (e.g., Chemin et al. 2004; Gentile et al. 2004, 2005; Kuzio de Naray et al. 2006; Simon et al. 2005; Swaters et al. 2003b). By their nature, these highresolution two-dimensional velocity fields eliminate concerns about long-slit placement, and highlight the presence of noncircular motions. They also typically probe in detail the innermost regions of the galaxies where the cusp-core conflict is most severe. Even with these improved observations, the data remain more consistent with cored halos and tend to have concentrations too low for $\Lambda$ CDM (Kuzio de Naray et al. 2006; Gentile et al. 2007; but see Swaters et al. 2003b).

In a previous paper (Kuzio de Naray et al. 2006, hereafter K06) , we presented high-resolution optical velocity fields and derived rotation curves for 11 LSB galaxies, along with pseudoisothermal (core) and NFW (cusp) halo fits in the limit of no baryons. LSB galaxies are thought to be dark matter-dominated down to small radii (de Blok \& McGaugh 1996, 1997; Boriello \& Salucci 2001; but see Fuchs 2003) with the light simply providing a tracer for the dark matter, so neglecting the baryons is not entirely unreasonable. The stellar mass 
contribution in these systems is low, which reduces errors involving the uncertainty in the stellar mass-to-light ratio, $\Upsilon_{*}$, and in turn, the isolation of the dark matter component. While halo fits are often made under the assumption that all observed rotation is due to the dark matter, it is not strictly true. In this paper, we present halo fits for four assumptions about $\Upsilon_{*}$ for the galaxies in K06 with available photometry. We also present observed DensePak velocity fields, rotation curves and halo fits for six new galaxies, along with additional DensePak observations, updated rotation curves and halo fits for three galaxies in K06.

In $\S 2$ we describe our sample of galaxies and the DensePak observations; the data reduction is discussed in $\S 3$. The observed velocity fields and derived rotation curves are presented in $\S 4$ and the zero disk halo fits are presented in $\S 5$. Mass models for the galaxies with photometry are presented in $\S 6$ and $\S 7$. A discussion of the mass model results is given in $\S 8$. Our conclusions are stated in $\S 9$.

\section{SAMPLE AND OBSERVATIONS}

During the nights of 2006 March 28-29, April 6-8, August 28-30 and September 25-27 we observed 17 LSB galaxies using the DensePak Integrated Field Unit (IFU) on the $3.5 \mathrm{~m} \mathrm{WIYN}^{3}$ telescope at the Kitt Peak National Observatory (KPNO). UGC 4325, DDO 64, and F583-1 were observed during March and April. These observations augment previous DensePak observations published in K06. The 14 targets that were observed in August and September were selected primarily from the Nearby Galaxies Catalogue (Tully 1988). Selection criteria for these galaxies included positions satisfying $18^{h} \lesssim \alpha \lesssim$ $08^{h}$ and $10^{\circ} \lesssim \delta \lesssim 50^{\circ}$, inclinations between $30^{\circ}$ and $85^{\circ}$, heliocentric velocities $\lesssim 3000 \mathrm{~km} \mathrm{~s}^{-1}$, and an estimated $V_{\text {flat }}$ (approximated by $V_{\text {flat }} \sim 0.5 W_{20}(\sin \mathrm{i})^{-1}$ ) between roughly $50 \mathrm{~km} \mathrm{~s}^{-1}$ and $100 \mathrm{~km} \mathrm{~s}^{-1}$. Additionally, we selected those galaxies which appear to have diffuse $\mathrm{H} \alpha$ emission and lack indicators of significant noncircular motions (e.g., strong bars or gross asymmetries). We also targeted galaxies with previous long-slit rotation curves or $\mathrm{H} \alpha$ imaging.

Because of inclement weather and telescope scheduling, we did not have $\mathrm{H} \alpha$ imaging or long-slit rotation curves for most of the galaxies in the sample prior to making the DensePak observations. Without these data, there is no way of knowing how much $\mathrm{H} \alpha$ emission will be detected by the IFU until the observations are actually made. Thus, our observed sample of galaxies is signallimited; the IFU fibers detected sufficient $\mathrm{H} \alpha$ emission to create a useable velocity field in just under $50 \%$ of our target galaxies.

The observing setup and procedure were identical to that used in K06. The IFU orientation on the sky and the total number of pointings per galaxy were tailored to each galaxy so that the critical central regions were covered by the DensePak fibers. Each exposure was $1800 \mathrm{~s}$, and two exposures were taken at each pointing. To provide wavelength calibration, a $\mathrm{CuAr}$ lamp was observed

\footnotetext{
3 Based on observations obtained at the WIYN Observatory. The WIYN Observatory is a joint facility of the University of Wisconsin-Madison, Indiana University, Yale University, and the National Optical Astronomy Observatory.
}

before and after each pointing. We used the 860 line $\mathrm{mm}^{-1}$ grating in second order, centered near $\mathrm{H} \alpha$, giving a $58 \mathrm{~km} \mathrm{~s}^{-1}$ velocity resolution. The DensePak fibers are $3^{\prime \prime}$, however, we achieved $\sim 2^{\prime \prime}$ resolution by shifting the DensePak array by small amounts so that the spaces between the fibers were observed. The distances to the galaxies in the sample are such that a $3^{\prime \prime}$ fiber provides subkiloparsec resolution.

\section{DATA REDUCTION}

The data were reduced following the procedure described in K06. Briefly, the observations were reduced in IRAF $^{4}$ using the HYDRA package. Night-sky emission lines (Osterbrock et al. 1996) were used for wavelength calibration, and velocities were measured by fitting Gaussians to both the sky lines and the four galactic emission lines of interest: $\mathrm{H} \alpha,[\mathrm{N}$ II] $\lambda 6584,[\mathrm{~S} \mathrm{II}] \lambda 6717$ and $[\mathrm{S}$ II $] \lambda 6731$. The average error on individual emissionline velocities due to centroiding accuracies was roughly $1.5 \mathrm{~km} \mathrm{~s}^{-1}$. The velocity assigned to each fiber was the arithmetic mean of the measured emission-line velocities in the fiber. The error on the fiber velocity was the maximum difference between the measured velocities and the mean. Many of these errors were less than $5 \mathrm{~km} \mathrm{~s}^{-1}$, although a few were as high as $\sim 20 \mathrm{~km} \mathrm{~s}^{-1}$. If only $\mathrm{H} \alpha$ was observed in a fiber, the observed $\mathrm{H} \alpha$ velocity was taken as the fiber velocity. Without other lines to determine the maximum difference, we adopt $10 \mathrm{~km} \mathrm{~s}^{-1}$ as a conservative error estimate based on experience with long-slit data (e.g. McGaugh et al. 2001).

Using the input shifts at the telescope, individual DensePak pointings were combined to construct the observed velocity field. An $\mathrm{H} \alpha$ flux image of the galaxy constructed from the DensePak observations was compared to an actual $\mathrm{H} \alpha$ image of the galaxy obtained at the KPNO $2 \mathrm{~m}$ telescope to confirm the accuracy of the offsets. The fiber maps were registered to the galaxy image by the correspondence of flux maxima through the fibers to observed features like individual Hit regions. We find the accuracy of the fiber positions $\left(\sim 0^{\prime \prime} .6\right)$ to be consistent with our results in K06. Both large $\left(1^{\prime \prime}-2^{\prime \prime}\right)$ and small $\left(\sim 0^{\prime \prime} .7\right)$ shifts can be made confidently.

Rotation curves were derived from the observed velocity fields using the tilted-ring fitting program ROTCUR (Begeman 1989) following the procedure outlined in K06. To construct a rotation curve, ROTCUR requires the systemic velocity, inclination, kinematic center, and position angle of the galaxy in addition to the observed fiber velocities. For a more complete description of ROTCUR as applied here, the reader is referred to K06.

Because the DensePak data cover the centers of the galaxies and probe the regime of solid-body rotation, neither the galaxy center nor the inclination could be determined from the observations. The velocity field centers were therefore fixed to the optical centers of the galaxies, as determined by the centroid of ellipses fit in the surface photometry, and the inclination was fixed to published values (Tully 1988; de Blok \& McGaugh 1996; McGaugh et al. 2001;

4 IRAF is distributed by the National Optical Astronomy Observatory, which is operated by the Association of Universities for Research in Astronomy (AURA), Inc., under agreement with the National Science Foundation. 
Mass Models of LSB Galaxies

TABLE 1

Properties of Observed Galaxies

\begin{tabular}{|c|c|c|c|c|c|c|c|c|c|c|c|}
\hline $\begin{array}{c}\text { Galaxy } \\
\text { (1) }\end{array}$ & $\begin{array}{c}\text { R.A. } \\
(\mathrm{J} 2000.0) \\
(2)\end{array}$ & $\begin{array}{c}\text { Decl. } \\
(\mathrm{J} 2000.0) \\
(3)\end{array}$ & $\begin{array}{c}\mu_{0}(\mathrm{~B}) \\
\left(\operatorname{mag}_{(4)}^{\operatorname{arcsec}}{ }^{-2}\right) \\
(4)\end{array}$ & $\begin{array}{l}\text { Distance } \\
(\mathrm{Mpc}) \\
(5)\end{array}$ & $\begin{array}{c}i \\
(\mathrm{deg}) \\
(6)\end{array}$ & $\begin{array}{c}V_{\text {hel }} \\
\left(\mathrm{km} \mathrm{s}^{-1}\right) \\
(7)\end{array}$ & $\begin{array}{c}R_{\max } \\
(\mathrm{kpc}) \\
(8)\end{array}$ & $\begin{array}{c}V_{\max } \\
\left(\mathrm{km} \mathrm{s}^{-1}\right) \\
(9)\end{array}$ & $\begin{array}{l}\text { P.A. } \\
\text { (deg) } \\
(10)\end{array}$ & $\begin{array}{c}\sigma \\
\left(\mathrm{km} \mathrm{s}^{-1}\right) \\
(11)\end{array}$ & $\begin{array}{l}\text { References } \\
\text { (12) }\end{array}$ \\
\hline UGC 4325 & 081920.5 & +500035 & $22.5^{a}$ & 10.1 & 41 & 514 & 2.9 & 110 & 52 & 9.0 & $2,2,2$ \\
\hline DDO 64 & 095022.4 & +312916 & $\ldots$ & 6.1 & 60 & 517 & 1.9 & 60 & 97 & 7.9 & $2,2,2$ \\
\hline F583-1 & 155727.5 & +203958 & 24.1 & 32 & 63 & 2256 & 5.8 & 72 & 355 & 8.7 & $4,4,4$ \\
\hline NGC $7137^{b}$ & 214813.0 & +220934 & $20.7^{c}$ & 22.5 & 38 & 1669 & 3.2 & 62 & 44 & 7.1 & $1,10,12$ \\
\hline UGC 11820 & 214928.4 & $\begin{array}{r}+141352 \\
\end{array}$ & 23.7 & 13.3 & 50 & 1088 & 2.9 & 93 & 309 & 8.9 & $14,7,9$ \\
\hline UGC 128 & 001350.9 & $\begin{array}{r}+355939 \\
\end{array}$ & 24.2 & 60 & 57 & 4509 & 13.5 & 133 & 62 & 15.5 & $8,3,3$ \\
\hline UGC 191 & $\begin{array}{lll}00 & 20 & 05.2\end{array}$ & +105248 & 22.7 & 17.6 & 39 & 1139 & 2.4 & 97 & 156 & 7.7 & $14,13,12$ \\
\hline UGC 1551 & 020337.5 & +240432 & 22.5 & 20.2 & 63 & 2663 & 3.8 & 83 & 114 & 9.7 & $5,11,12$ \\
\hline NGC $959^{d}$ & $0232 \quad 23.9$ & +352941 & $21.9^{e}$ & 7.8 & 51 & 590 & 1.6 & 77 & 64 & 8.7 & $6,7,7$ \\
\hline
\end{tabular}

Noте. - Units of right ascension are hours, minutes, and seconds, units of declination are degrees, arcminutes, and arcseconds. Col.(1): Galaxy

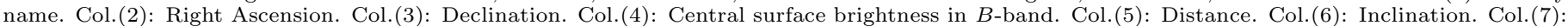

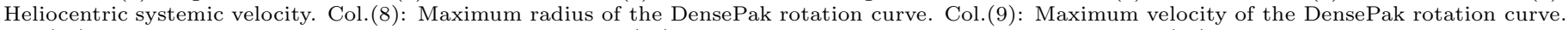

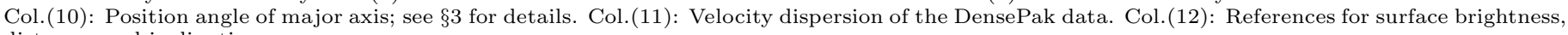
distance, and inclination.

${ }^{a}$ Converted from $R$ band assuming $B-R=0.9$.

${ }^{b}$ NGC $7137=$ UGC 11815.

${ }^{c}$ Converted from $V$ band assuming $B-V=0.57$.

${ }^{d}$ NGC $959=$ UGC 2002.

${ }^{e}$ Converted from $I$ band assuming $V-I=0.90$ and $B-V=0.53$

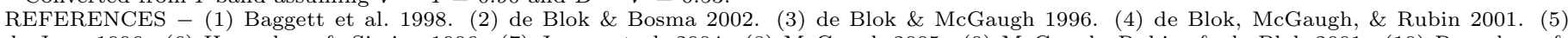

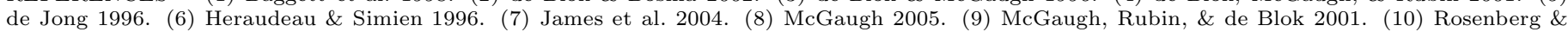
Schneider 2003. (11) Swaters \& Balcells 2002. (12) Tully 1988. (13) van Zee \& Haynes 2006. (14) van Zee et al. 1997.

de Blok et al. 2001; de Blok \& Bosma 2002; James et al. 2004). The systemic velocities were determined by ROTCUR. We used ROTCUR to determine the position angle of the major axis, using published long-slit values as the initial guess. If the position angle could not be wellconstrained by ROTCUR, then it was fixed to the longslit value. The position angles of UGC 4325, DDO 64, and F583-1 were not well-constrained by ROTCUR, and remained fixed at the values used in K06. The position angle of the major axis was well-constrained by ROTCUR for all of the galaxies in the new sample except for UGC 128. In the case of UGC 128, the position angle was set to the position angle of the Hi velocity field of van der Hulst et al. (1993). For reasons described in K06, we did not impose a minimum error on the rotation curve points. However, we added in quadrature to the error bars on the final ROTCUR rotation curve the velocity error from Gaussian centroiding accuracy, typically $\sim 1.5 \mathrm{~km} \mathrm{~s}^{-1}$, corrected for inclination. We have also not corrected the rotation velocities for asymmetric drift, as the corrections are typically only $\sim 2 \mathrm{~km} \mathrm{~s}^{-1}$ (see also de Blok \& Bosma 2002).

\section{OBSERVED VELOCITY FIELDS AND DERIVED ROTATION CURVES}

In this section, we present the DensePak fiber positions, observed velocity fields, and rotation curves in Figures 1-3. A description of each galaxy is given, and the properties of the galaxies for which a rotation curve was derived are listed in Table 1.

\subsection{Extended Observations of Previously Observed Galaxies}

Additional DensePak observations were made of three galaxies published in K06. In this section, we describe the positions of the new DensePak pointings, present the augmented velocity fields, and discuss the resulting changes in the rotation curves.
$\boldsymbol{U G C}$ 4325 - There were four new DensePak pointings roughly through the galaxy center from SE to NW. All eight pointings are shown on the $\mathrm{H} \alpha$ image (Figure 1) of the galaxy. The fiber velocities were the average of the $\mathrm{H} \alpha$ and $[\mathrm{S} \mathrm{II}] \lambda 6717$ lines. The position angle of the major axis remained fixed at the average of the position angles of previous long-slit observations de Blok \& Bosma 2002; Swaters et al. 2003a). With the addition of the new DensePak pointings, the DensePak rotation curve extends to $\sim 60^{\prime \prime}$ and remains in excellent agreement with the long-slit $\mathrm{H} \alpha$ rotation curve of de Blok \& Bosma (2002).

DDO 64- There were two new DensePak pointings along the SE end of DDO 64. All five pointings are shown on the $\mathrm{H} \alpha$ image (Figure 1) of the galaxy. The fiber velocities were the average of the $\mathrm{H} \alpha$ and $[\mathrm{S} \mathrm{II}] \lambda 6717$ lines. The position angle remained fixed at the value determined in de Blok \& Bosma (2002). With the addition of the new DensePak pointings and an $\mathrm{H} \alpha$ image, the positions of the DensePak fibers on the galaxy have been updated and the center of the velocity field has moved $13^{\prime \prime} \mathrm{E}$ and $2^{\prime \prime} .5 \mathrm{~S}$. The new DensePak rotation curve is now consistent with the long-slit $\mathrm{H} \alpha$ rotation curve of de Blok \& Bosma (2002) in the inner 20". Beyond 20", the DensePak rotation curve shows a more linear rise with less scatter than its previous version.

F583-1 - There were two new DensePak pointings, one to the north and one to the east, on this galaxy. All three pointings are shown on the $R$-band image (Figure 1 ) of the galaxy from de Blok et al. (1996). The fiber velocities were the average of the $\mathrm{H} \alpha,[\mathrm{S} \mathrm{II}] \lambda 6717$, and [S II] $\lambda 6731$ lines. The receding side of the galaxy and more of the minor axis are now covered with the additional pointings. The position angle remained fixed at the value listed in McGaugh et al. (2001). The new DensePak rotation curve shows less scatter than the curve derived from a single DensePak pointing, and it displays a higher level of consistency with the long-slit $\mathrm{H} \alpha$ 
rotation curve of de Blok, McGaugh, \& Rubin (2001).

\subsection{New Observations}

Of the 14 galaxies observed, there were eight galaxies for which meaningful velocity fields could not be constructed. ${ }^{5}$ The $\mathrm{H} \alpha$ emission in these galaxies was too faint to be detected and/or not spread out enough across the fiber array. Velocity fields and rotation curves were derived for the remaining six galaxies in the sample, and each is described below.

NGC 7137 - There were three DensePak pointings for this galaxy. The pointings are shown on the $\mathrm{H} \alpha$ image (Figure 2) of the galaxy. Spiral arms are clearly visible in this galaxy. The fiber velocities were the average of the $\mathrm{H} \alpha$, [N II] $\lambda 6584$, [S II] $\lambda 6717$, and [S II] $\lambda 6731$ lines. $\mathrm{H} \alpha$ emission was abundant and the majority of fibers detected emission. The inclination was fixed to the value in Tully (1988). The position angle of the major axis was well-constrained by ROTCUR. The rotation curve rises steeply out to roughly $10^{\prime \prime}$ then dips slightly before rising again.

$\boldsymbol{U G C}$ 11820 - This galaxy is both large and diffuse on the sky. There is a central concentration of $\mathrm{H} \alpha$ emission, which is perhaps a bar, that runs roughly NE-SW and also two large, diffuse arms that extend from the end of the bar. There were three DensePak pointings along the central feature. The pointings are shown on the $\mathrm{H} \alpha$ image (Figure 2) of the galaxy. The fiber velocities were the average of the $\mathrm{H} \alpha$, [S II] $\lambda 6717$, and [S II] $\lambda 6731$ lines. The $\mathrm{H} \alpha$ emission was sparse and only roughly half of the fibers detected emission. The inclination was fixed to the value listed in McGaugh et al. (2001). The position angle of the major axis was well-constrained by ROTCUR.

UGC 128 - There were three DensePak pointings across this galaxy. The pointings are shown on the $\mathrm{H} \alpha$ image (Figure 2) of the galaxy. The fiber velocities were the average of the $\mathrm{H} \alpha,[\mathrm{N}$ II] $\lambda 6584$, and $[\mathrm{S} \mathrm{II}] \lambda 6717$ lines. The $\mathrm{H} \alpha$ emission in this galaxy was sparse, but the emission that was present was scattered across the three DensePak pointings such that both the approaching and receding sides of the velocity field were mapped. The position angle was fixed to the position angle of the Hi velocity field of van der Hulst et al. (1993) and the inclination was fixed to the value listed in de Blok \& McGaugh (1996). The DensePak rotation curve is plotted with the HI rotation curve of Verheijen \& de Blok (1999). The DensePak rotation curve is largely consistent with if not slightly steeper than the HI curve and does not go out far enough to show a clear turn-over.

$\boldsymbol{U G C} 191$ - There were two DensePak pointings for this galaxy and they are shown on the $\mathrm{H} \alpha$ image (Figure 3). The fiber velocities were the average of the $\mathrm{H} \alpha$, $[\mathrm{S} \mathrm{II}] \lambda 6717$, and $[\mathrm{SII}] \lambda 6731$ lines. There is ample $\mathrm{H} \alpha$ emission in the galaxy and almost every fiber had a detection. The inclination was fixed to the value in Tully (1988). The position angle of the major axis was wellconstrained by ROTCUR. The rotation curve rises linearly and has no clear turn-over.

$\boldsymbol{U G C} \mathbf{C 5 5 1}$ - There were three DensePak pointings for this galaxy. The pointings are shown on the $\mathrm{H} \alpha$ image (Figure 3) of the galaxy. The fiber velocities were the av-

5 F469-2, UGC 2034, UGC 2053, UGC 11944, UGC 12048/NGC 7292, UGC 12082, UGC 12212, and UGC 12632. erage of the $\mathrm{H} \alpha$, $[\mathrm{N}$ II] $\lambda 6584$, [S II] $\lambda 6717$, and $[\mathrm{S} \mathrm{II}] \lambda 6731$ lines. $\mathrm{H} \alpha$ emission was detected in nearly all of the fibers. The inclination was fixed to the value in Tully (1988). The position angle of the major axis was well-constrained by ROTCUR. There is a twist in the velocity field that is suggestive of the presence of a bar. Noncircular motions are probably important inside of $15^{\prime \prime}$ where the rotation curve is mostly flat. Beyond $15^{\prime \prime}$, there is a linear rise in the rotation curve.

NGC 959- There were three DensePak pointings across the length of this galaxy. The pointings are shown on the $\mathrm{H} \alpha$ image (Figure 3 ) of the galaxy. The fiber velocities were the average of the $\mathrm{H} \alpha$, [N II] $\lambda 6584$, $[\mathrm{S} \mathrm{II}] \lambda 6717$, and $[\mathrm{S} \mathrm{II}] \lambda 6731$ lines. $\mathrm{H} \alpha$ emission was abundant and the majority of fibers detected emission. The inclination was fixed to the value listed in James et al. (2004). The position angle of the major axis was well-constrained by ROTCUR. The rotation curve is wellbehaved with a steady rise and a turnover to $V_{\text {flat }} \sim 80$ $\mathrm{km} \mathrm{s}^{-1}$.

\section{ZERO DISK HALO FITS}

In this section, we present the pseudoisothermal and NFW halo fits to the DensePak rotation curves in the zero disk case. By ignoring the velocity contribution from the baryons and attributing all rotation to dark matter, we are able to put an upper limit on the slope and/or concentration of the halo density profile. For those galaxies with photometry, halo fits for three assumptions about the stellar mass-to-light ratio are presented in $\S 6$.

\subsection{Halo Models}

The cuspy NFW halo and the cored pseudoisothermal halo are two of the most well-known competing descriptions of dark matter halos. We provide a brief description of each below.

\subsubsection{NFW Profile}

Numerical simulations show that the density of CDM halos rises steeply toward the halo center. The exact value of the inner slope of the CDM halo varies slightly depending on the simulation (e.g. Navarro et al. 1996, 1997; Moore et al. 1999; Reed et al. 2003; Navarro et al. 2004; Diemand et al. 2005). From an observational perspective, there is very little to distinguish the various flavors of cuspy CDM halos, and we choose to fit the data with the NFW halo.

The NFW mass-density distribution is described as

$$
\rho_{N F W}(R)=\frac{\rho_{i}}{\left(R / R_{s}\right)\left(1+R / R_{s}\right)^{2}},
$$

in which $\rho_{i}$ is related to the density of the universe at the time of halo collapse, and $R_{s}$ is the characteristic radius of the halo. The NFW rotation curve is given by

$$
V(R)=V_{200} \sqrt{\frac{\ln (1+c x)-c x /(1+c x)}{x[\ln (1+c)-c /(1+c)]}},
$$

with $x=R / R_{200}$. The rotation curve is parameterized by a radius $R_{200}$ and a concentration parameter $c=$ $R_{200} / R_{s}$, both of which are directly related to $R_{s}$ and $\rho_{i}$. Here $R_{200}$ is the radius at which the density contrast exceeds 200, roughly the virial radius; $V_{200}$ is the circular 

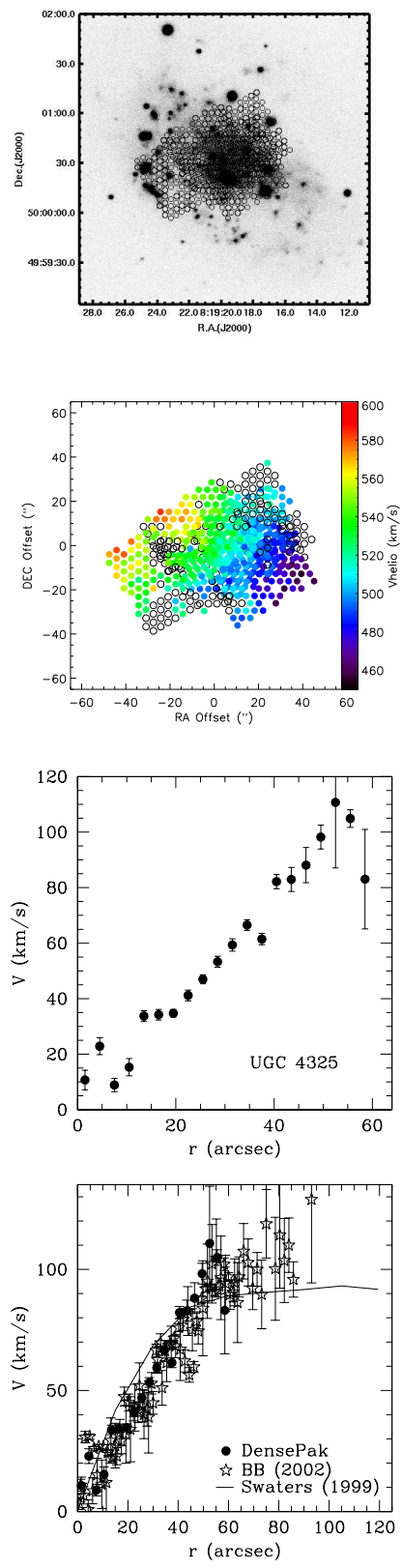
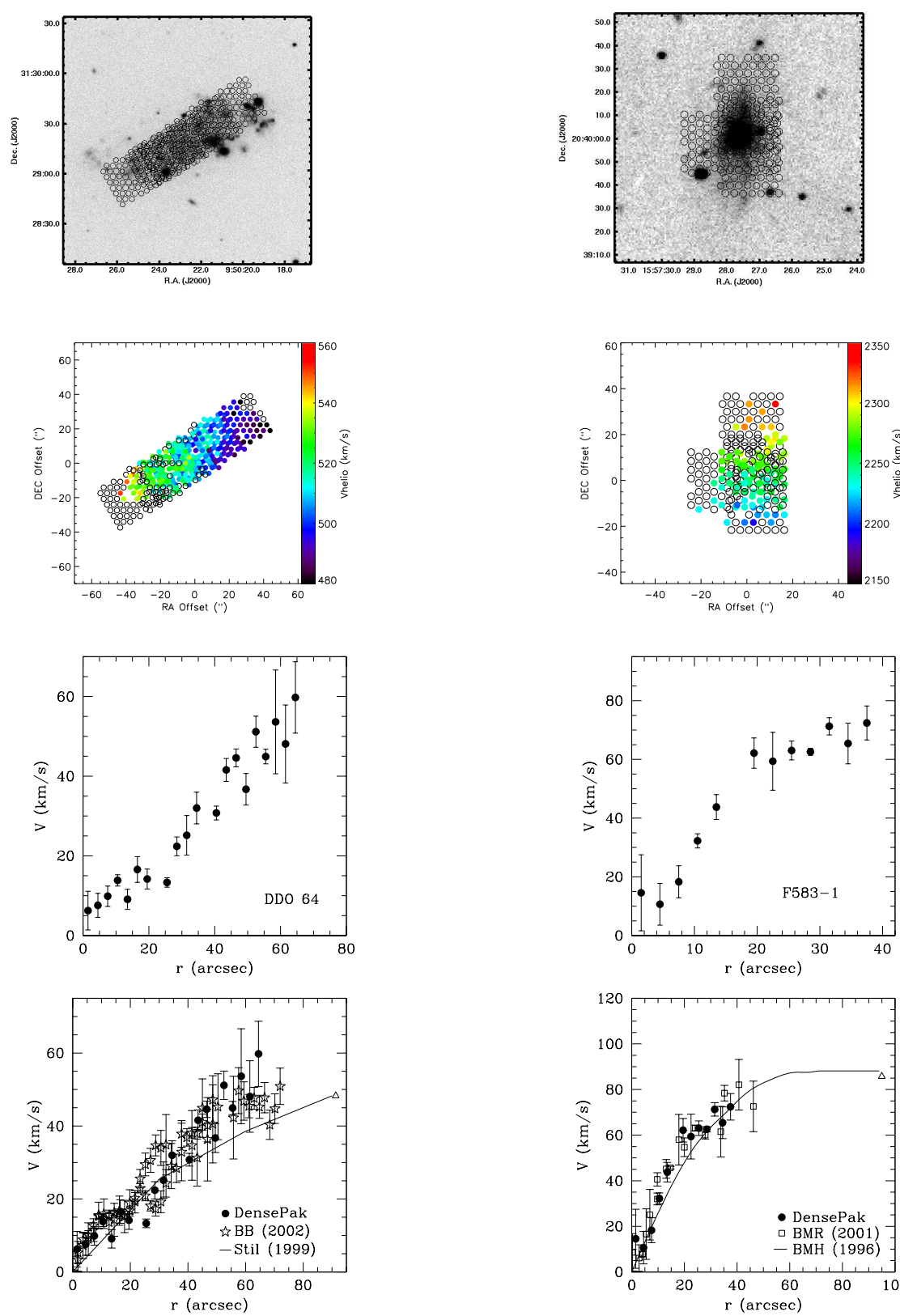
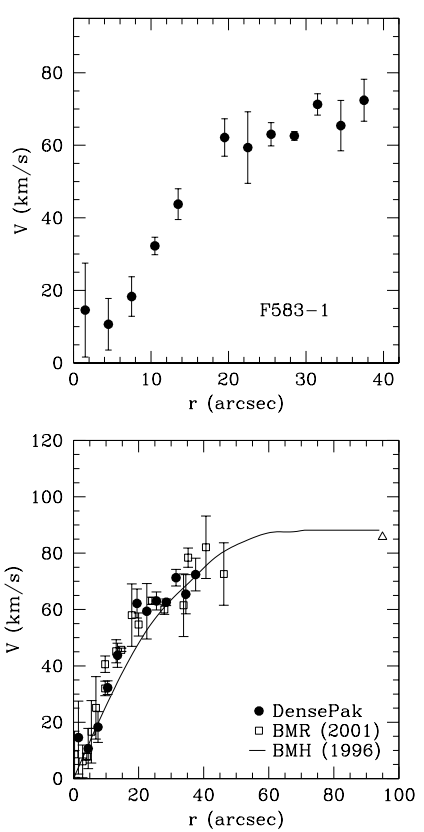

FIG. 1.- Additional observations of UGC 4325, DDO 64, and F583-1. Top row: Position of DensePak array on the H $\alpha$ images of UGC 4325 and DDO 64 and the $R$-band image of F583-1. Second row: Observed DensePak velocity field with new pointings. Empty fibers are those without detections. Third row: Updated DensePak rotation curves. Bottom row: Updated DensePak rotation curves plotted with long-slit $\mathrm{H} \alpha$ and Hi rotation curves. [See the electronic edition of the Journal for a color version of this figure.]

velocity at $R_{200}$ (Navarro et al. 1996, 1997). Because the NFW profile has a shallower slope than other cuspy halo models, it provides a lower limit on the slope of cuspy density profiles and, as such, gives the cuspy halo the best possible chance to fit the data.

\subsubsection{Pseudoisothermal Halo}

The pseudoisothermal halo describes a dark matter halo that has a core of roughly constant density. By construction, it produces flat rotation curves at large radii. The density profile of the pseudoisothermal halo is

$$
\rho_{i s o}(R)=\rho_{0}\left[1+\left(R / R_{C}\right)^{2}\right]^{-1},
$$

with $\rho_{0}$ being the central density of the halo and $R_{C}$ representing the core radius of the halo. The rotation curve corresponding to this density profile is

$$
V(R)=\sqrt{4 \pi G \rho_{0} R_{C}^{2}\left[1-\frac{R_{C}}{R} \arctan \left(\frac{R}{R_{C}}\right)\right]} .
$$

The pseudoisothermal halo is empirically motivated and predates halo profiles stemming from numerical simulations.

\subsection{Halo Fits to Previously Observed Galaxies}

We find the best-fit zero disk case isothermal and NFW halos to the new DensePak rotation curves of UGC 4325, 

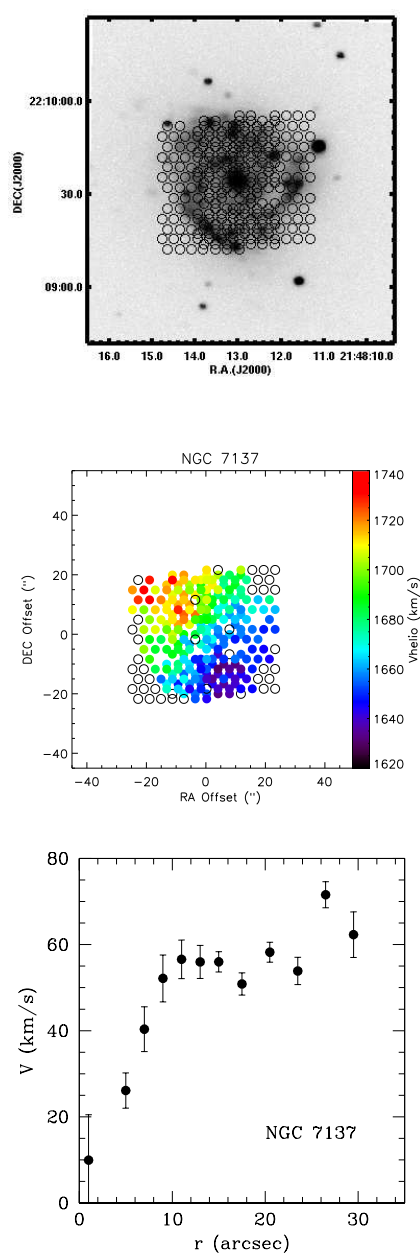
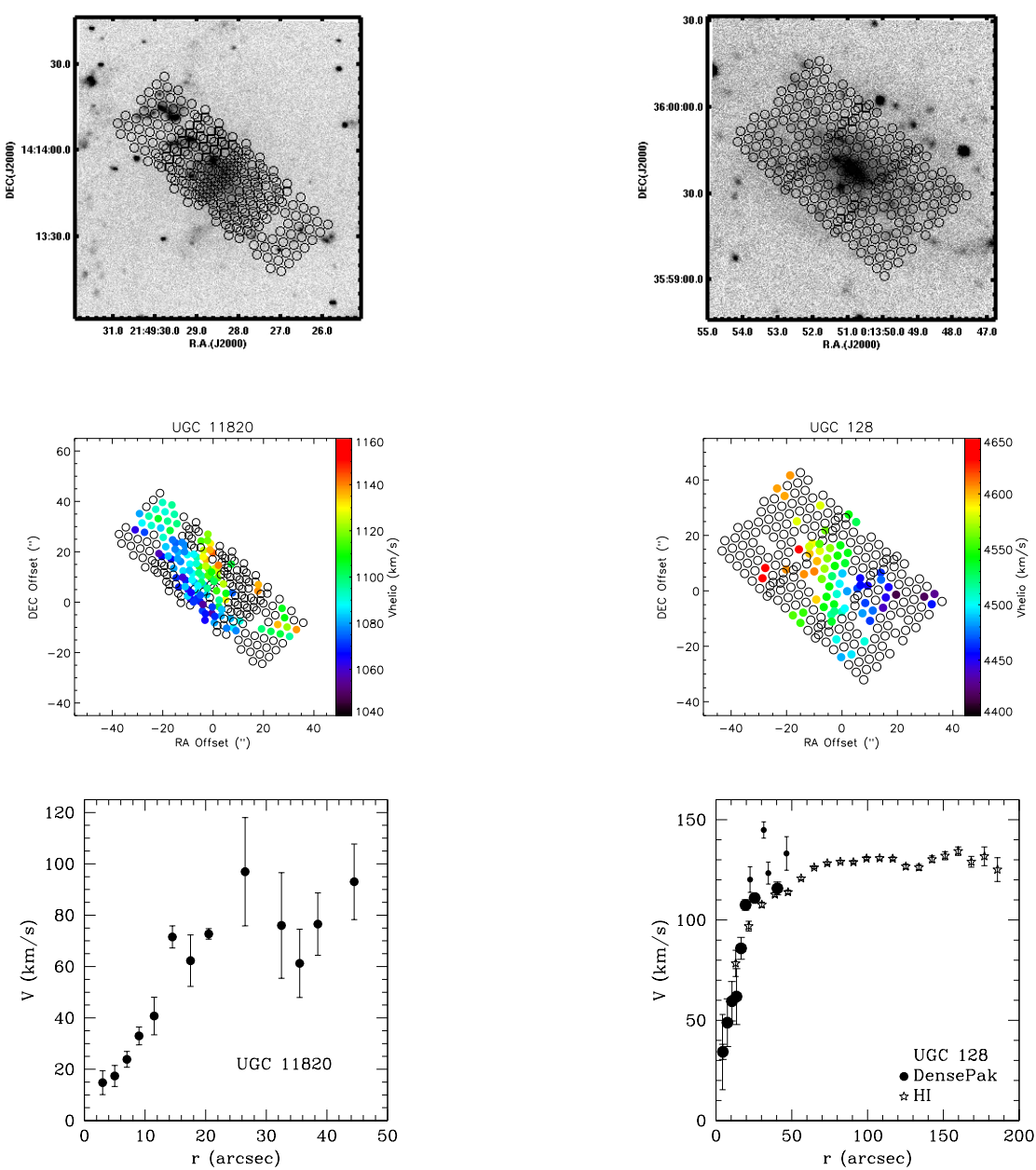

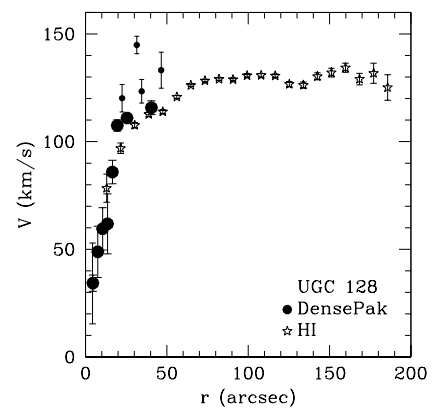

FIG. 2.- Results for NGC 7137, UGC 11820, \& UGC 128. Top row: Position of DensePak array on the H $\alpha$ images of the galaxies. Middle row: Observed DensePak velocity field. Empty fibers are those without detections. Bottom row: DensePak rotation curves. The UGC 128 DensePak points at large radii with $\sigma>4 \mathrm{~km} \mathrm{~s}^{-1}$ are plotted as smaller points. The DensePak rotation curve of UGC 128 is plotted with the Hi rotation curve of Verheijen \& de Blok (1999) (stars). [See the electronic edition of the Journal for a color version of this figure.]

DDO 64, and F583-1. When available, the DensePak rotation curves have been supplemented with previous smoothed long-slit $\mathrm{H} \alpha$ and Hi rotation curves. We use the entire long-slit rotation curve, and include only those Hi points that extend beyond the radial range of both the DensePak and long-slit data. Uncertainties from possible resolution effects are avoided by using only the outer $\mathrm{HI}$ points.

For these three galaxies, we also fit an NFW halo called $\mathrm{NFW}_{\text {constrained }}$ (hereafter $\mathrm{NFW}_{\text {constr }}$ ), as described in detail in K06. This halo fit is motivated by NFW fits that have parameters which are unrealistic or inconsistent with $\Lambda$ CDM. Briefly, the constrained halo was required to match the velocities at the outer radii of each galaxy while constraining the concentration to agree with cosmology. The concentrations were calculated using Equation (7) of de Blok et al. (2003), which gives the concentration as a function of of $V_{200}$ (Navarro et al. 1997), and then adjusted to the cosmology of Tegmark et al. (2004) by subtracting 0.011 dex (McGaugh, Barker, \& de Blok 2003 ).

The halo fits are plotted over the data in Figure 4, and the halo parameters are listed in Tables 2 and 3. For comparison, the numbers mentioned in the text below are the fits from $\mathrm{K} 06$, unless specifically noted otherwise.

$\boldsymbol{U G C}$ 4325 - The isothermal fit to UGC $4325 \mathrm{im}-$ proves slightly with the addition of the four new DensePak pointings $\left(R_{c}=3.3 \pm 0.2 ; \rho_{0}=91 \pm 4 ; \chi^{2}\right.$ $=3.8$ ). An unconstrained NFW halo still could not be fitted to the data, and the poor quality of the $\mathrm{NFW}_{\text {constr }}$ fit remains virtually unimproved $\left(c=6.9 ; V_{200}=249\right.$; $\left.\chi^{2}=40\right)$. This galaxy remains best-described by the isothermal halo.

DDO 64-The two new DensePak pointings on DDO 64 help to improve the quality of both the isothermal and $\mathrm{NFW}_{\text {constr }}$ fits. The values of the isothermal halo parameters remain within the errors of the original values, but the value of $\chi_{r}^{2}$ decreases $\left(R_{c}=4.4 \pm 0.9 ; \rho_{0}=38 \pm 3\right.$; $\left.\chi^{2}=5.5\right)$. While no unconstrained NFW halo fit could be made, the quality of the $\mathrm{NFW}_{\text {constr }}$ halo significantly improves $\left(c=9.2 ; V_{200}=62 ; \chi^{2}=20\right)$. This does not mean, however, that the NFW halo is a good fit to the data. The NFW constr halo continues to overshoot the data at radii interior to where it was forced to match the 

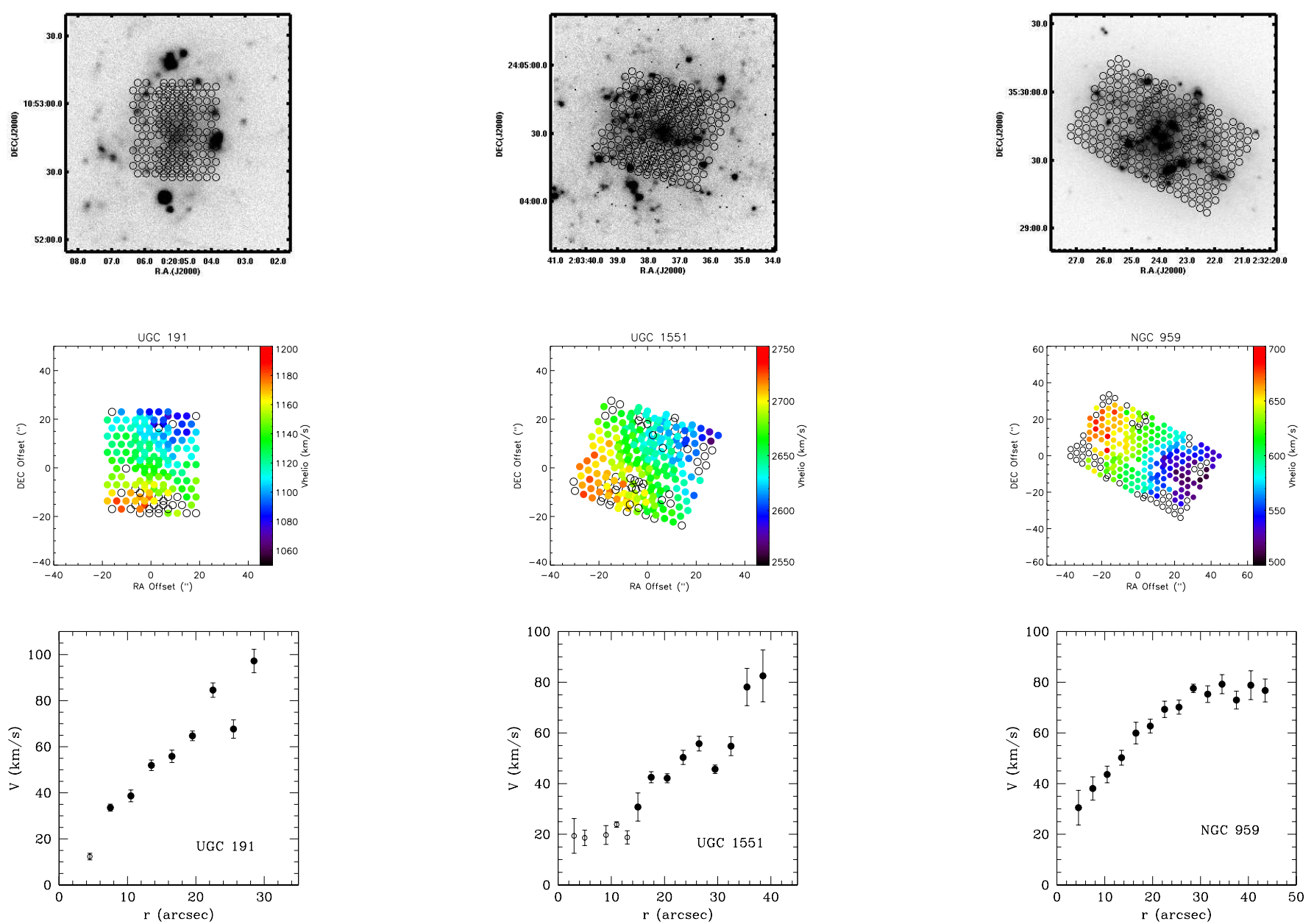

FIG. 3.- Results for UGC 191, UGC 1551, \& NGC 959. Top row: Position of DensePak array on the H $\alpha$ images of the galaxies. Middle row: Observed DensePak velocity field. Empty fibers are those without detections. Bottom row: DensePak rotation curves. The open points in the UGC 191 and UGC 1551 rotation curves were excluded from the halo fits. [See the electronic edition of the Journal for a color version of this figure.]

data. While it passes through the large errorbars on the inner rotation curve points, significant noncircular motions would need to be important all the way out to $\sim 1$ $\mathrm{kpc}$ in order to boost the observed velocities up to the expected NFW velocities.

F583-1 - The isothermal and NFW fits are significantly better constrained by the addition of the two new DensePak pointings. F583-1 remains best-described by the isothermal halo. The values of the halo parameters remain within the errors of the previous values, but the value of $\chi_{r}^{2}$ falls from 5.4 to $0.5\left(R_{c}=2.7 \pm 0.1 ; \rho_{0}=35\right.$ \pm 2 ). The new values of the isothermal halo parameters are almost indistinguishable from the values determined by de Blok et al. (2001): $R_{c}=2.44 \pm 0.06, \rho_{0}=33.0 \pm$ 1.1. The values of the parameters of the unconstrained NFW fit are also mostly unchanged, and $\chi_{r}^{2}$ decreases to 1.7 from $8.7\left(c=4.7 \pm 0.7 ; V_{200}=133 \pm 21\right)$. The value of the concentration, $c=4.5$, is still too low to be consistent with $\Lambda$ CDM. The value of $\chi_{r}^{2}$ also drops for the $\mathrm{NFW}_{\text {constr }}$ fit $\left(\chi^{2}=11 \rightarrow 6.5\right)$, but the $\mathrm{NFW}$ velocities continue to over-predict the observed velocities interior to where they were forced to match the data.

\subsection{Halo Fits to New Observations}

We find the best-fit zero disk case isothermal and NFW halos to the DensePak rotation curves of NGC 7137, UGC 11820, UGC 128, UGC 191, UGC 1551, and NGC 959. In the case of UGC 128, we combine the DensePak rotation curve with the Hi rotation curve of Verheijen \& de Blok (1999), using only those Hi points beyond the radial range of the DensePak data. We do not make an $\mathrm{NFW}_{\text {constr }}$ fit to these six galaxies, as the radial range of the data does not extend beyond the rising part of the rotation curve. In Figure 4 we plot the halo fits over the data, and list the halo parameters in Tables 2 and 3.

NGC 7137 - The isothermal halo is a slightly better fit to NGC 7137 than the NFW halo, but the difference is not particularly significant. There are "bumps and wiggles" in the rotation curve that simple, smooth halo models cannot fit. The value of the concentration, $c=$ 15 , for the NFW fit is on the high side of values expected in a $\Lambda \mathrm{CDM}$ cosmology.

UGC 11820 - UGC 11820 is fit relatively well by the isothermal halo; no NFW halo could be fit to the rotation 

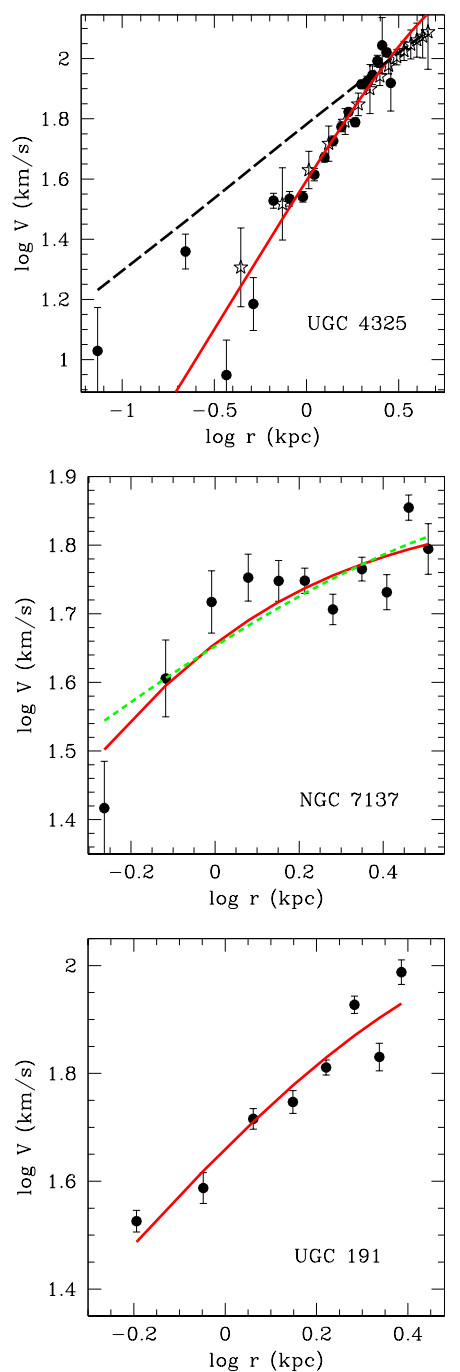
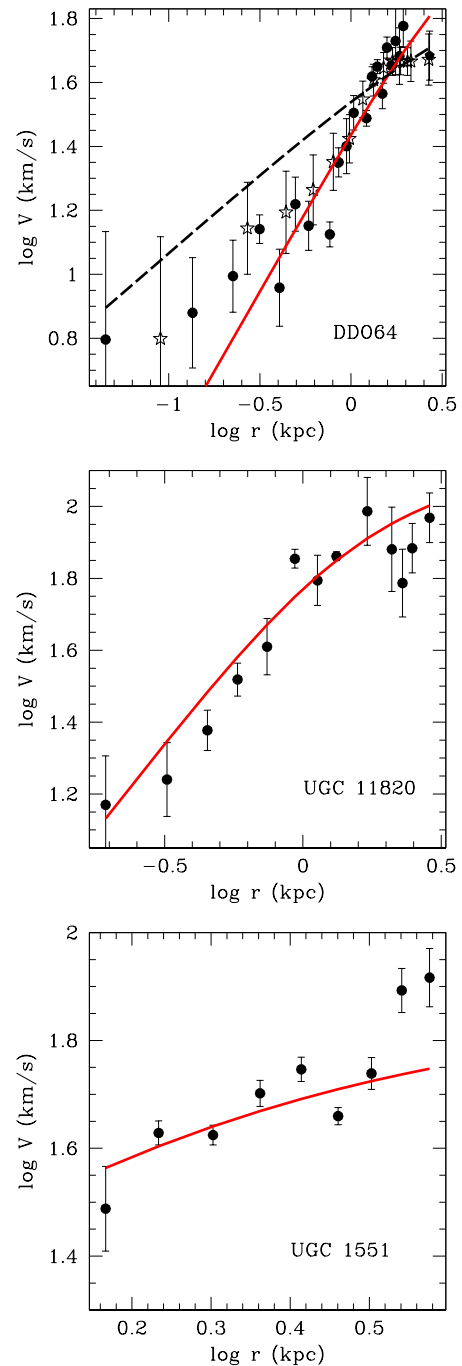
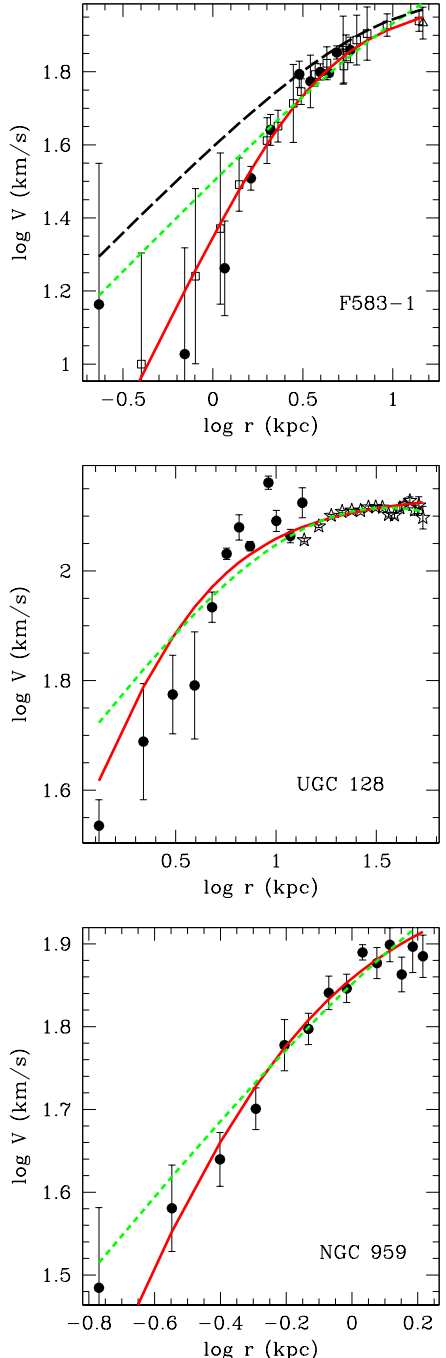

FIG. 4. - Halo fits to the DensePak rotation curves. The red solid line is the best-fit isothermal halo, the green short-dashed line is the best-fit unconstrained NFW halo, and the black long-dashed line is the best-fit NFW constr halo. NFW $\mathrm{Nonstr}_{\text {fits }}$ were only made to UGC 4325, DDO 64, and F583-1. [See the electronic edition of the Journal for a color version of this figure.]

curve.

$\boldsymbol{U G C} 128$ - UGC 128 is more consistent with the isothermal halo than the NFW halo, though the value of $\chi_{r}^{2}$ is high for both fits and both halo models overestimate the rotation velocities at small radii. The bestfitting concentration, $c=8.9$, is reasonable for a galaxy of this size in a $\Lambda$ CDM cosmology. The best-fitting isothermal halo parameters for the combined DensePak $+\mathrm{HI}$ rotation curve are $R_{c}=2.3 \pm 0.1$ and $\rho_{0}=66 \pm 0.6$. de Blok \& McGaugh (1996) also fit an isothermal halo to the van der Hulst et al. (1993) Hi rotation curve and find $R_{c}=4.0$ and $\rho_{0}=21.7$. The differences in the halo parameters reflect the inclusion of the slightly steeper DensePak rotation curve.

$\boldsymbol{U G C} 191$ - We exclude the innermost point of the UGC 191 rotation curve due to its unrealistically small formal error bar and the steep jump in velocity between it and the next rotation curve point. Excluding this point from the halo fits does not significantly alter the values of the halo parameters, but does improve the values of the reduced $\chi^{2}$. No NFW fit could be made to the DensePak rotation curve. The data were fit with an isothermal halo, albeit with a large $\chi_{r}^{2}$. The UGC 191 DensePak rotation curve is linearly rising and shows no turn-over over in the radial range covered by the DensePak data. To be useful for distinguishing between halo models, data points at larger radii are needed to better constrain the halo fits.

$\boldsymbol{U} \boldsymbol{G} \boldsymbol{C} 1551$ - We exclude the first five points of the UGC 1551 rotation curve because the twist in the velocity field suggests that a bar may be present in the galaxy. An NFW fit could not be made to the data; an isothermal fit was made, but was not well-constrained. Like UGC 191, data at larger radii are necessary for obtaining useful constraints on the halo models.

NGC 959- NGC 959 is well described by the isothermal halo and is a slightly better fit than the NFW halo. The value of the concentration, $c=23$, of the best fit NFW halo is on the high side of expected values for galaxies in a $\Lambda \mathrm{CDM}$ cosmology. 
Mass Models of LSB Galaxies

TABLE 2

ISOThERMAL HALO PARAmeters

\begin{tabular}{|c|c|c|c|c|c|c|c|c|}
\hline \multirow[b]{2}{*}{ Galaxy } & \multicolumn{4}{|c|}{ ZERO DISK } & \multicolumn{4}{|c|}{ MINIMAL DISK } \\
\hline & $R_{c}$ & $\rho_{0}$ & $\chi_{r}^{2}$ & $\overline{\Upsilon_{*}}$ & $\overline{R_{c}}$ & $\rho_{0}$ & $\chi_{r}^{2}$ & $\overline{\Upsilon_{*}}$ \\
\hline UGC 4325 & $4.1 \pm 0.3$ & $88 \pm 3$ & 3.2 & 0.0 & $4.6 \pm 1.5$ & $77 \pm 5$ & 3.1 & 0.57 \\
\hline F563-V2 & $1.5 \pm 0.1$ & $119 \pm 6$ & 0.71 & 0.0 & $1.4 \pm 0.2$ & $114 \pm 20$ & 0.68 & 0.44 \\
\hline F563-1 & $2.1 \pm 0.1$ & $67 \pm 2$ & 0.43 & 0.0 & $2.0 \pm 0.2$ & $61 \pm 10$ & 0.48 & 0.68 \\
\hline DDO 64 & $3.3 \pm 0.5$ & $43 \pm 3$ & 3.2 & 0.0 & $4.1 \pm 3.8$ & $37 \pm 6$ & 3.3 & 0.62 \\
\hline F568-3 & $3.8 \pm 0.2$ & $27 \pm 1$ & 1.2 & 0.0 & $4.0 \pm 0.5$ & $22 \pm 3$ & 1.5 & 0.66 \\
\hline UGC 5750 & $5.7 \pm 0.4$ & $7.1 \pm 0.3$ & 0.83 & 0.0 & $6.5 \pm 1.1$ & $5.2 \pm 0.6$ & 0.84 & 0.62 \\
\hline NGC 4395 & $0.7 \pm 0.1$ & $258 \pm 9$ & 2.9 & 0.0 & $0.57 \pm 0.05$ & $318 \pm 42$ & 2.7 & 0.70 \\
\hline F583-4 & $1.3 \pm 0.1$ & $67 \pm 2$ & 0.67 & 0.0 & $1.2 \pm 0.2$ & $66 \pm 16$ & 0.62 & 0.53 \\
\hline F583-1 & $2.5 \pm 0.1$ & $30 \pm 2$ & 0.50 & 0.0 & $2.4 \pm 0.2$ & $30 \pm 3$ & 0.59 & 0.62 \\
\hline NGC 7137 & $0.6 \pm 0.1$ & $274 \pm 17$ & 3.1 & 0.0 & $\ldots$ & $\cdots$ & $\ldots$ & $\ldots$ \\
\hline UGC 11820 & $1.1 \pm 0.1$ & $274 \pm 21$ & 2.9 & 0.0 & $\ldots$ & $\ldots$ & $\ldots$ & $\ldots$ \\
\hline UGC 128 & $2.3 \pm 0.1$ & $66 \pm 0.6$ & 8.5 & 0.0 & $\ldots$ & $\ldots$ & $\ldots$ & $\ldots$ \\
\hline UGC 191 & $1.7 \pm 0.1$ & $138 \pm 10$ & 5.8 & 0.0 & $\ldots$ & $\ldots$ & $\ldots$ & $\ldots$ \\
\hline UGC 1551 & $1.3 \pm 0.2$ & $57 \pm 5$ & 5.5 & 0.0 & $\ldots$ & $\ldots$ & $\ldots$ & $\ldots$ \\
\hline \multirow[t]{2}{*}{ NGC 959} & $0.4 \pm 0.1$ & $1117 \pm 29$ & 1.2 & 0.0 & $\cdots$ & $\cdots$ & $\cdots$ & $\cdots$ \\
\hline & \multicolumn{4}{|c|}{ POPSYNTH } & \multicolumn{4}{|c|}{ MAXIMUM DISK } \\
\hline Galaxy & $R_{c}$ & $\rho_{0}$ & $\chi_{r}^{2}$ & $\Upsilon_{*}$ & $R_{c}$ & $\rho_{0}$ & $\chi_{r}^{2}$ & $\Upsilon_{*}$ \\
\hline $\mathrm{UGC} 4325^{a}$ & $6.2 \pm 3.3$ & $66 \pm 5$ & 3.2 & 1.14 & 2.5 & 45 & 11 & 4.5 \\
\hline F563-V2 & $1.4 \pm 0.2$ & $102 \pm 20$ & 0.75 & 0.88 & $4.7 \pm 5.9$ & $7.1 \pm 8.8$ & 1.4 & 4.0 \\
\hline \multirow[t]{2}{*}{$\mathrm{F} 563-1^{b}$} & $2.1 \pm 0.2$ & $54 \pm 10$ & 0.50 & 1.36 & $4.2 \pm 1.2$ & $13 \pm 6$ & 0.83 & 6.5 \\
\hline & $\begin{array}{l}2.1+0.2 \\
\ldots\end{array}$ & $\ldots$ & $\ldots$ & $\ldots$ & $12.5 \pm 9.0$ & $2.7 \pm 1.6$ & 1.3 & 10.0 \\
\hline DDO $64^{a}$ & $5.9 \pm 11.1$ & $32 \pm 5$ & 3.5 & 1.24 & 1.5 & 24 & 2.2 & 5.0 \\
\hline F568-3 & $4.7 \pm 0.8$ & $16 \pm 3$ & 1.8 & 1.32 & $6.1 \pm 1.8$ & $10 \pm 3$ & 2.5 & 2.3 \\
\hline UGC 5750 & $7.5 \pm 1.6$ & $4.1 \pm 0.5$ & 0.95 & 1.24 & $9.8 \pm 3.2$ & $2.6 \pm 0.5$ & 1.3 & 2.2 \\
\hline NGC $4395^{a}$ & $0.50 \pm 0.04$ & $355 \pm 50$ & 2.8 & 1.40 & 19 & 0.32 & 4.1 & 9.0 \\
\hline F583-4 $4^{a}$ & $1.2 \pm 0.2$ & $63 \pm 16$ & 0.62 & 1.06 & 8 & 1.4 & 1.2 & 10.0 \\
\hline \multirow[t]{2}{*}{$\mathrm{F} 583-1^{b}$} & $2.5 \pm 0.2$ & $27 \pm 3$ & 0.60 & 1.24 & $3.6 \pm 0.5$ & $14 \pm 2$ & 0.83 & 5.0 \\
\hline & $\ldots$ & $\ldots$ & $\ldots$ & $\ldots$ & $6.9 \pm 1.7$ & $5.2 \pm 0.9$ & 2.0 & 10.0 \\
\hline
\end{tabular}

Note. $-R_{c}$ is in kpc; $\rho_{0}$ is in $10^{-3} \mathrm{M}_{\odot} \mathrm{pc}^{-3}$. Photometry is unavailable for NGC $7137, \mathrm{UGC} 11820, \mathrm{UGC}$ 128, UGC 191, UGC 1551, \& NGC 959; halo fits beyond zero disk are not presented.

${ }^{a}$ The baryons can account for most of the velocity in the maximum disk fit. See text for details of fit.

${ }^{b}$ As discussed in the text, F563-1 and F583-1 have two possible values of $\Upsilon_{* \text { Max }}$.

\subsection{Summary of Zero Disk Halo Fits}

In $\S 5.2$ and $\S 5.3$ we have presented updated zero disk halo fits to three galaxies from $\mathrm{K} 06$ and six new galaxies observed with DensePak. Overall, we find the isothermal halo to be a better description of seven of these galaxies than the NFW halo. Two of the new galaxies do not have data at large enough radii to put useful constraints on the halo models. When NFW fits could be made, the concentrations were often beyond the range of values expected for $\Lambda$ CDM. We find that the parameters of the halo fits to the three previously observed galaxies do not significantly change, but are better constrained by the additional DensePak coverage. The DensePak rotation curves of the six new galaxies could all be fit by the isothermal halo, and an NFW fit could be made to only three. Only one of the three NFW fits had a concentration consistent with the range expected for galaxies in a $\Lambda \mathrm{CDM}$ cosmology; the other two are on the high end of expected values. We also find that the quality of both the isothermal and NFW halo fits is greatly improved when the radial range of the data extends into the flat part of the rotation curve. Of the six new galaxies, only NGC 959 has a DensePak rotation curve which clearly turns over and flattens, and it is this galaxy that has the isothermal and NFW fits with the lowest $\chi_{r}^{2}$.

\section{MASS MODELS}

Low surface brightness galaxies are dark matterdominated down to small radii. Because of this, the velocity contribution from the baryons is often disregarded when fitting dark matter halo models to the galaxy rotation curves. This type of fit which ignores the contribution of the stars and gas, such as in $\S 5$, is usually called the minimum-disk case. While the dark matter is the dominant mass component of LSB galaxies at all radii, baryons are still important. To accurately determine the distribution of the dark matter, it is necessary to properly account for the stars and gas in the galaxies and their contribution to the observed rotation. The velocity component coming from the stars is determined from the surface photometry scaled by the stellar mass-to-light ratio, $\Upsilon_{*}$. This is a straightforward computation of the gravitational potential of the observed stars. However, the true value of $\Upsilon_{*}$ is difficult to determine, and as discussed below, can be assigned a value following a number of techniques. Similarly, the velocity component from the gas is determined using Hi surface density profiles. The conversion from observed $21 \mathrm{~cm}$ luminosity to atomic gas mass is well understood from the physics of the spin-flip transition. A scaling factor, the inverse of the hydrogen mass fraction, is usually included to account for the helium and metals also present in the galaxies.

In $\S 5$ of this paper and in K06, we have so far ignored the baryons in the halo fits to LSB galaxies observed with DensePak. For those galaxies with $R$-band photometry 
TABLE 3

NFW Halo Parameters

\begin{tabular}{|c|c|c|c|c|c|c|c|c|}
\hline \multirow[b]{2}{*}{ Galaxy } & \multicolumn{4}{|c|}{ ZERO DISK } & \multicolumn{4}{|c|}{ MINIMAL DISK } \\
\hline & $\mathrm{c}$ & $V_{200}$ & $\chi_{r}^{2}$ & $\Upsilon_{*}$ & $\mathrm{c}$ & $V_{200}$ & $\chi_{r}^{2}$ & $\Upsilon_{*}$ \\
\hline UGC 4325 & 6.9 & 249 & 39 & 0.0 & 1.0 & 1002 & 12 & 0.57 \\
\hline $\mathrm{F} 563-\mathrm{V} 2$ & $7.7 \pm 2.0$ & $128 \pm 32$ & 0.40 & 0.0 & $8.4 \pm 1.5$ & $105 \pm 17$ & 0.46 & 0.44 \\
\hline F563-1 & $7.8 \pm 1.3$ & $106 \pm 10$ & 0.88 & 0.0 & $7.6 \pm 1.3$ & $100 \pm 9$ & 0.89 & 0.68 \\
\hline DDO 64 & 9.2 & 62 & 12 & 0.0 & 1.0 & 376 & 6.5 & 0.62 \\
\hline F568-3 & 8.2 & 110 & 12 & 0.0 & 1.0 & 465 & 3.9 & 0.66 \\
\hline UGC 5750 & $0.5 \pm 0.1$ & $320 \pm 43$ & 1.7 & 0.0 & 1.0 & 167 & 1.7 & 0.62 \\
\hline NGC 4395 & $10.1 \pm 0.6$ & $77 \pm 4$ & 2.1 & 0.0 & $11.5 \pm 1.0$ & $63 \pm 4$ & 2.1 & 0.70 \\
\hline F583-4 & $5.5 \pm 2.2$ & $92 \pm 32$ & 0.41 & 0.0 & $5.7 \pm 1.4$ & $83 \pm 18$ & 0.41 & 0.53 \\
\hline F583-1 ${ }^{a}$ & $4.5 \pm 0.8$ & $120 \pm 20$ & 1.7 & 0.0 & $5.1 \pm 1.2$ & $102 \pm 21$ & 1.8 & 0.62 \\
\hline NGC 7137 & $15 \pm 3$ & $56 \pm 10$ & 3.4 & 0.0 & $\ldots$ & $\ldots$ & $\ldots$ & $\ldots$ \\
\hline UGC 11820 & & & & & $\cdots$ & $\cdots$ & $\cdots$ & $\cdots$ \\
\hline UGC 128 & $8.9 \pm 0.2$ & $111 \pm 0.7$ & 9.3 & 0.0 & $\ldots$ & $\ldots$ & $\ldots$ & $\ldots$ \\
\hline UGC 191 & $\ldots$ & $\ldots$ & $\ldots$ & $\ldots$ & $\cdots$ & $\ldots$ & $\cdots$ & $\ldots$ \\
\hline UGC 1551 & $\ldots$ & . & $\ldots$ & $\ldots$ & $\ldots$ & $\ldots$ & $\ldots$ & $\ldots$ \\
\hline \multirow[t]{2}{*}{ NGC 959} & $23 \pm 4$ & $76 \pm 15$ & 1.7 & 0.0 & $\cdots$ & $\cdots$ & $\cdots$ & $\cdots$ \\
\hline & \multicolumn{4}{|c|}{ POPSYNTH } & \multicolumn{4}{|c|}{ MAXIMUM DISK } \\
\hline Galaxy & $\mathrm{c}$ & $V_{200}$ & $\chi_{r}^{2}$ & $\Upsilon_{*}$ & $\mathrm{c}$ & $V_{200}$ & $\chi_{r}^{2}$ & $\Upsilon_{*}$ \\
\hline UGC 4325 & 1.0 & 897 & 12 & 1.14 & 1.0 & 283 & 12 & 4.5 \\
\hline F563-V2 & $7.8 \pm 1.7$ & $104 \pm 20$ & 0.52 & 0.88 & 1.0 & 203 & 1.1 & 4.0 \\
\hline \multirow[t]{2}{*}{$\mathrm{F} 563-1^{b}$} & $7.0 \pm 1.3$ & $102 \pm 11$ & 0.90 & 1.36 & 1.0 & 283 & 1.0 & 6.5 \\
\hline & & & & & 1.0 & 210 & 1.5 & 10.0 \\
\hline DDO 64 & 1.0 & 332 & 6.4 & 1.24 & 1.0 & 146 & 2.6 & 5.0 \\
\hline F568-3 & 1.0 & 404 & 4.3 & 1.32 & 1.0 & 317 & 5.2 & 2.3 \\
\hline UGC 5750 & 1.0 & 141 & 1.9 & 1.24 & 1.0 & 101 & 2.4 & 2.2 \\
\hline NGC 4395 & $12.5 \pm 1.1$ & $61 \pm 4$ & 2.2 & 1.40 & $\ldots$ & $\ldots$ & $\ldots$ & 9.0 \\
\hline F583-4 & $5.8 \pm 1.5$ & $86 \pm 19$ & 0.40 & 1.06 & $\ldots$ & .. & $\ldots$ & 10.0 \\
\hline \multirow[t]{2}{*}{$\mathrm{F} 583-1^{b}$} & $4.9 \pm 1.2$ & $110 \pm 26$ & 1.9 & 1.24 & 1.0 & 298 & 2.1 & 5.0 \\
\hline & & & $\ldots$ & & 1.0 & 173 & 3.3 & 10.0 \\
\hline
\end{tabular}

Note. $-V_{200}$ is in $\mathrm{km} \mathrm{s}^{-1}$. Italicized halo parameters are forced fits. See text for details. Photometry is unavailable for NGC 7137, UGC 11820, UGC 128, UGC 191, UGC 1551, \& NGC 959; halo fits beyond zero disk are not presented.

${ }^{a}$ The parameters of the updated $N F W_{\text {constr }}$ fit for the zero disk case of F583-1 are $c=8.7, V_{200}=83, \chi_{r}^{2}$ $=6.5$.

${ }^{b}$ As discussed in the text, F563-1 and F583-1 have two possible values of $\Upsilon_{* \text { Max }}$.

TABLE 4

Galaxy Parameters

\begin{tabular}{lclcc}
\hline \hline \multicolumn{1}{c}{ Galaxy } & $h$ (kpc) & $(B-R)$ & $\Upsilon_{*}($ Pop $)$ & References \\
\hline UGC 4325 & 1.6 & 0.85 & 1.14 & 1,3 \\
F563-V2 & 2.1 & $0.51^{a}$ & 0.88 & 2,4 \\
F563-1 & 2.8 & 0.96 & 1.36 & 2,5 \\
DDO 64 & 1.2 & $0.9^{b}$ & 1.24 & 1 \\
F568-3 & 4.0 & 0.94 & 1.32 & 2,5 \\
UGC 5750 & 5.6 & $0.9^{b}$ & 1.24 & 2,2 \\
NGC 4395 & 2.3 & $\ldots^{c} c$ & 1.40 & 1 \\
F583-4 & 2.7 & 0.8 & 1.06 & 2,6 \\
F583-1 & 1.6 & 0.9 & 1.24 & 2,6 \\
& & & &
\end{tabular}

Note. - Col.(2): Scale length (kpc). Col.(4): $\Upsilon_{*}$ for the popsynth case as determined from the colors. Col.(5): References for $h$ and $(B-R)$, respectively: (1) de Blok \& Bosma (2002) (2) de Blok, McGaugh, \& Rubin (2001) (3) van den Bosch \& Swaters (2001) (4) de Blok \& McGaugh (1997) (5) de Blok, van der Hulst, \& Bothun (1995) (6) de Blok, McGaugh, \& van der Hulst (1996).

${ }^{a}(B-V)$ color

${ }^{b}$ Multicolor photometry unavailable; assuming $(B-R)=0.9$ for dwarf galaxies.

${ }^{c}$ Multicolor photomety unavailable; assuming $\Upsilon_{*}=1.4$.

and Hi surface density profiles we now include the contribution from the baryons and present the dark matter halo fits for three determinations of $\Upsilon_{*}$.

\subsection{Dynamical Components}

There are three separate components in galaxy mass models that contribute to the observed velocities: the stars, the gas, and the dark matter. The stars, gas, and dark matter are added together in quadrature to obtain the total velocity, $V_{\text {total }}^{2}=\Upsilon_{*} v_{*}^{2}+V_{\text {gas }}^{2}+V_{D M}^{2}$. In this section, we describe each of these dynamical components.

\subsubsection{Stars}

Galaxy photometry in combination with a stellar massto-light ratio, $\Upsilon_{*}$, is used to determine the stellar contribution to the observed galaxy rotation. It is difficult to determine the true value of $\Upsilon_{*}$ of a galaxy because it depends on many factors ranging from the initial mass function to the extinction. To address this uncertainty, we consider four different scenarios for the value of $\Upsilon_{*}$. We assume that $\Upsilon_{*}$ is constant with radius.

Zero disk - In this limiting case we ignore the contribution of the stars $\left(\Upsilon_{*}=0\right)$ and gas and attribute all rotation to dark matter. This case is typically called 'minimum disk' in the literature; we have chosen to refer to this case as zero disk because it aptly describes the omission of the baryons. The zero disk case puts an upper limit on the slope and/or concentration of the halo density profile. The results for the zero disk case were presented in $\S 5$ and also K06 (where they were referred to as minimum-disk) and are reproduced in Tables 2 and 

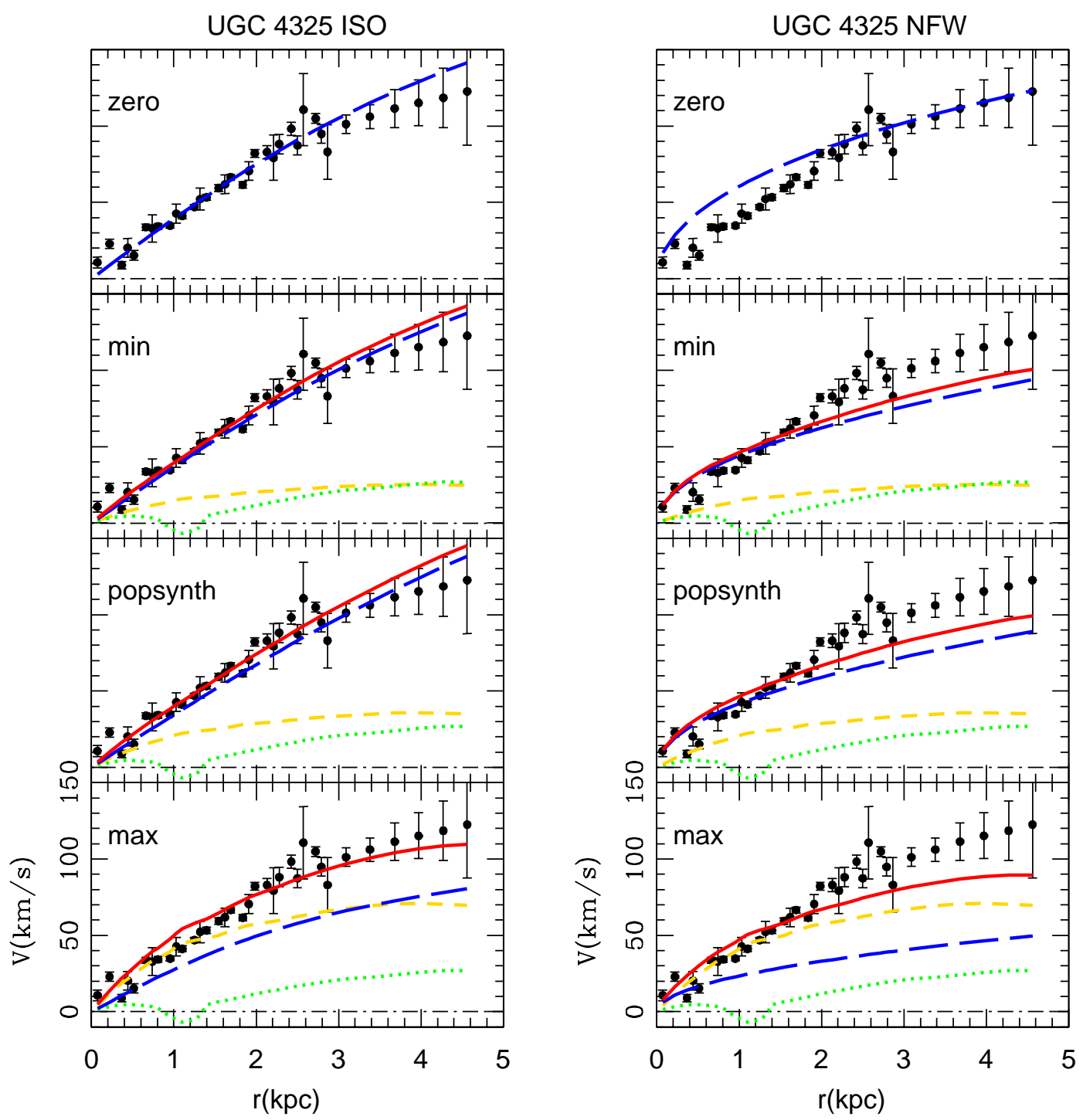

FIG. 5.- Isothermal and NFW halo fits for UGC 4325. The green dotted line is the rotation curve of the gas disk, the gold short-dashed line is the rotation curve of the stellar disk, the blue long-dashed line is the rotation curve of the dark matter halo, and the red solid line is the total model curve. The velocity contribution from both the stars and gas is ignored in the zero disk case. The minimal disk case considers the gas contribution and assumes a lightweight IMF for the stars. The popsynth case includes the gas contribution and uses population synthesis models to determine the stellar contribution. The maximum disk case includes the gas contribution and the stellar mass-to-light ratio is scaled up as far as the data will allow. [See the electronic edition of the Journal for a color version of this figure.]

3 for completeness. Two galaxies from K06, UGC 1281 and UGC 477, are excluded from mass models beyond zero disk because of their high inclinations and possible associated line-of-sight integration effects. Photometry is unavailable for the six new galaxies presented in $\S 5$; mass models beyond zero disk will not be presented.

Minimal disk - Though commonly employed, the zero disk case is unphysical. We next consider a more realistic minimal contribution of the stars and the gas. The $\Upsilon_{*}$ is determined from population synthesis models (see below) and then scaled by 0.5 (see McGaugh 2005) to simulate a lightweight IMF.

Popsynth-Here $\Upsilon_{*}$ is determined using the population synthesis models of Bell et al. (2003). Specifically, we use the relation between $\Upsilon_{*}$ and color as defined in their Table 7. For a scaled Salpeter IMF, $(B-R)$ and $(B-V)$ colors are related to $\Upsilon_{*}$. This is our best estimate of the baryonic mass from the perspective of stellar populations. The popsynth model also includes the gas contribution. Colors and corresponding $\Upsilon_{*}$ are listed in Table 4.

Maximum disk - We also consider the case where the $\Upsilon_{*}$ is scaled up as far as the data will allow. In high surface brightness galaxies, this approach usually fits the inner rotation curve well. In LSB galaxies, the shape of the stellar rotation curve is often not well-matched to the observed rotation curve. We have chosen a $\Upsilon_{*}$ that allows the stellar rotation curve to match the inner rotation curve points as well as possible, sometimes overshooting the innermost point in order to hit the next few. These 

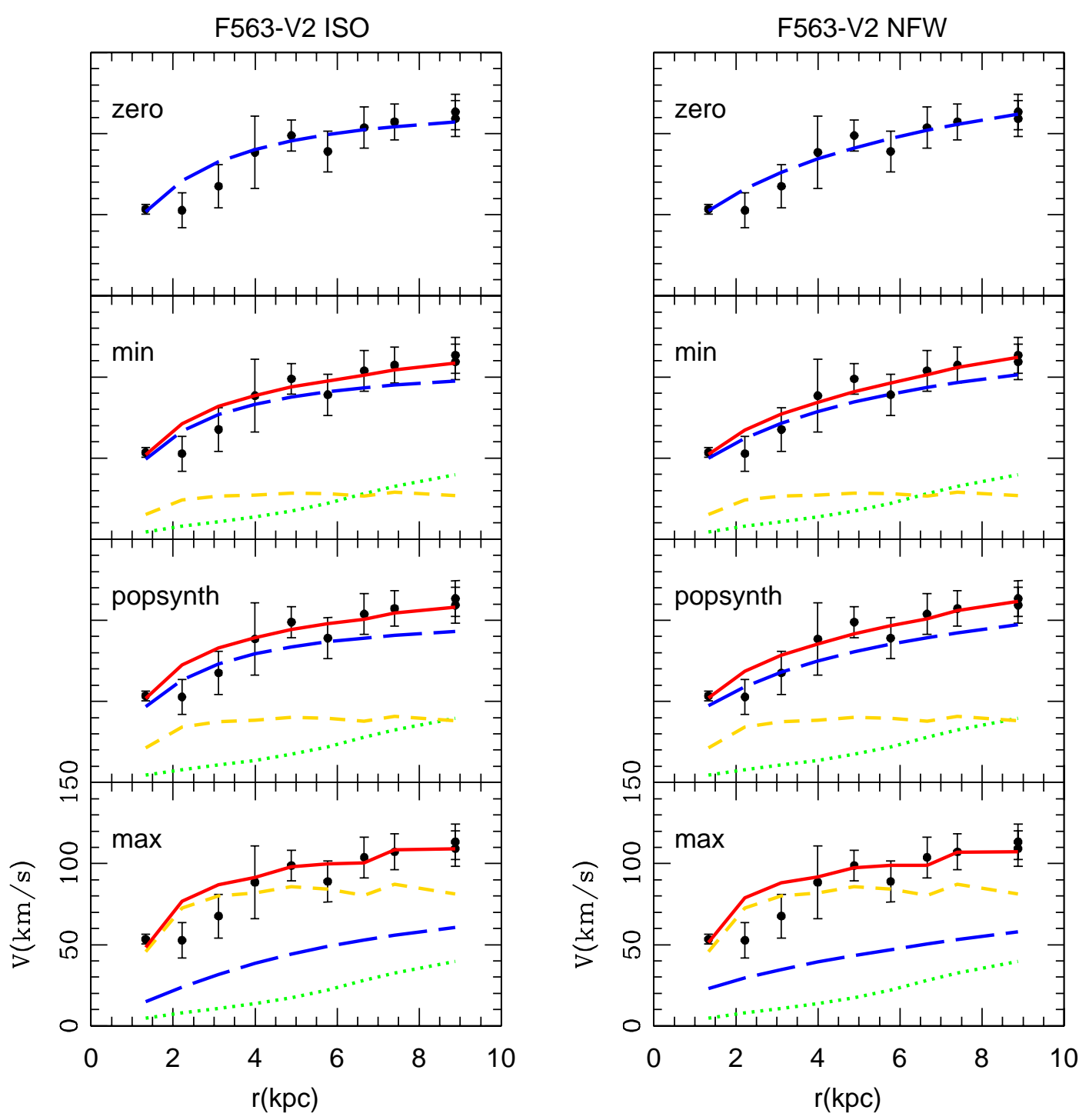

FIG. 6. - Isothermal and NFW halo fits for F563-V2. Line types are described in Figure 5. [See the electronic edition of the Journal for a color version of this figure.]

$\Upsilon_{*}$ are usually higher than those determined from the popsynth models, and in some cases (eg. F583-4) are substantially higher.

To model the stellar disk we have used the $R$-band photometry presented in de Blok et al. (1995), [Stil (1999), Swaters (1999), and Swaters et al. (2002). The GIPSY task ROTMOD was used to determine the rotation of the disk assuming a vertical $\operatorname{sech}^{2}$ distribution with a scale height $z_{0}=h / 6$ (van der Kruit \& Searle 1981). The stellar rotation curve computed from the photometry was resampled at the same radii as the combined DensePak+long-slit+Hi rotation curves.

Each estimator of $\Upsilon_{*}$ has its advantages and disadvantages. The zero disk $\Upsilon_{*}$ is often adopted and is useful because it provides an upper limit on the dark matter. Unfortunately, it is an unphysical assumption. Population synthesis models best represent what we know about stars, however, sometimes the data will allow a larger $\Upsilon_{*}$. And although $\Upsilon_{* M A X}$ often seems too large with respect to $\Upsilon_{* P O P}$, disk features like bars and spiral arms require a large disk mass (McGaugh \& de Blok 1998; Fuchs 2003) and support high $\Upsilon_{* M A X}$.

\subsubsection{Gas}

The gas present in galaxies also contributes to the observed galaxy rotation. HI is the dominant gas component, but to include helium and metals, the Hi data were scaled by a factor of 1.4. Substantial amounts of molecular gas are not obviously present in LSB galaxies (de Blok \& van der Hulst 1998; (Schombert et al. 1990). The total mass of $\mathrm{H}_{2}$ is almost certainly much less than that of Hi (Mihos, Spaans, \& McGaugh 1999). Moreover, in brighter galaxies $\mathrm{H}_{2}$ is known to trace the stars (Regan et al. 2001), so at most represents a slight tweak to $\Upsilon_{*}$. The contribution of the gas to the observed velocity is considered in the minimal, popsynth, and max- 

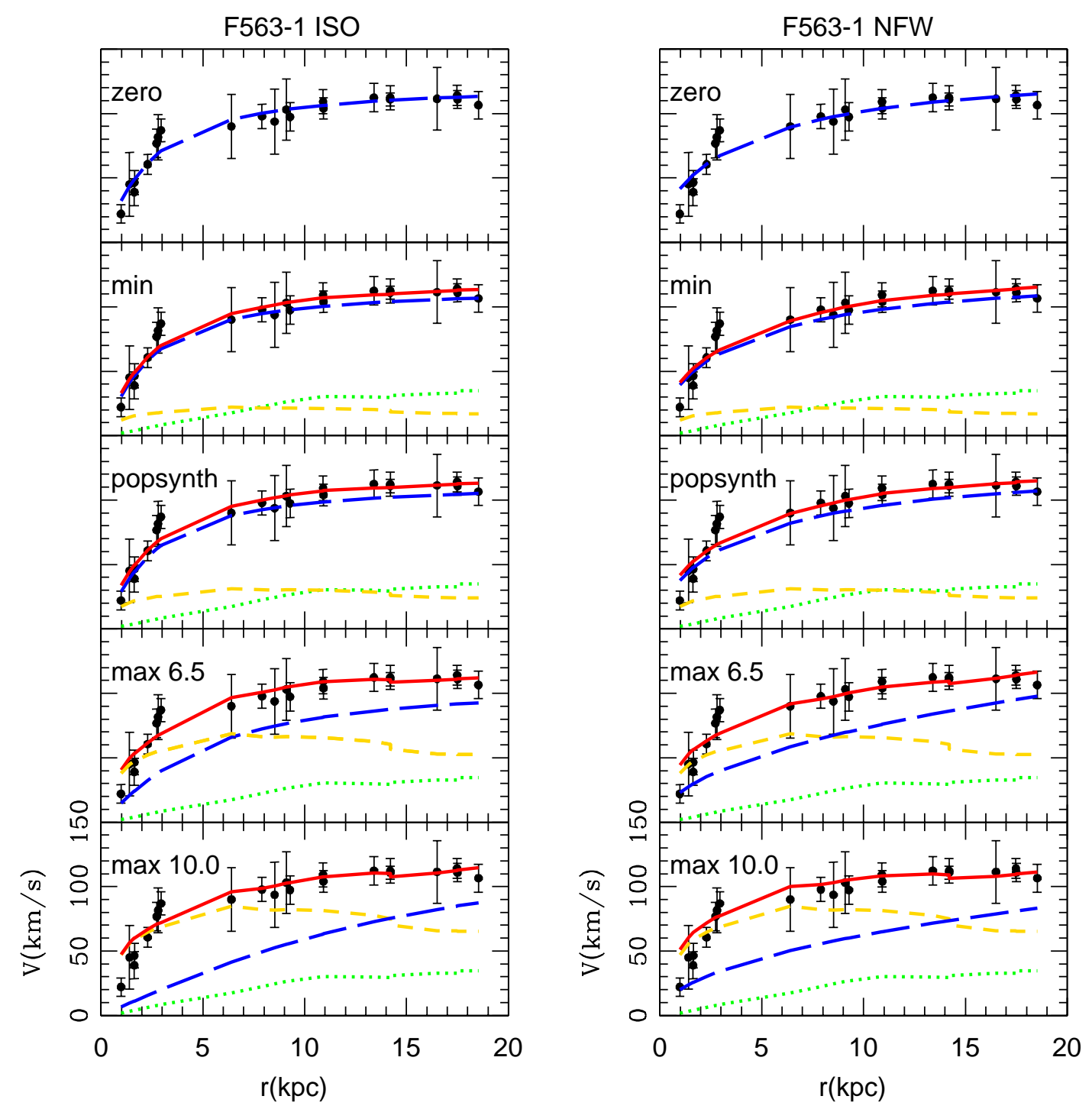

FIG. 7.- Isothermal and NFW halo fits for F563-1. Line types are described in Figure 5. [See the electronic edition of the Journal for a color version of this figure.]

imum disk cases.

The Hi surface density profiles presented in de Blok et al. (1996), van der Hulst et al. (1993), and Swaters (1999) were used to model the gas disk. The GIPSY task ROTMOD was used to determine the rotation of the disk assuming a thin disk. The gas rotation curve was resampled at the same radii as the combined DensePak+long-slit + Hi rotation curves.

\subsubsection{Dark Matter Halo}

In LSB galaxies there is usually a considerable amount of observed rotation unaccounted for after subtracting off the velocity of the stellar and gas disks, even in the maximum disk case. The remaining rotation is usually attributed to dark matter. While there have been a number of dark matter models proposed in the literature, we fit two of the most prominent competing profiles: the pseudo-isothermal halo and the NFW profile. Both halo profiles are described in $\S 5.1$.

\section{MASS MODEL RESULTS FOR INDIVIDUAL GALAXIES}

In this section, we present the isothermal and NFW halo fits for the four scenarios of $\Upsilon_{*}$. The halo parameters are listed in Tables 2 and 3. Figures 5-13 plot the halo fits over the data.

As $\Upsilon_{*}$ increases and the baryons become responsible for more of the observed velocity, less room is available for dark matter and the NFW halo becomes increasingly difficult to fit to the data. The best-fit concentrations drop to very small, and sometimes negative, values. In these cases, we forced an NFW fit with $c=1.0$. The halo parameters of forced fits are italicised in the tables.

$\boldsymbol{U G C}$ 4325 - UGC 4325 is clearly best described by an isothermal halo; an NFW halo could not be fit to the data for any $\Upsilon_{*}$ scenario. With $\Upsilon_{* M A X}=4.5$, the stellar rotation curve is able to describe the data well out to a radius of $\sim 1.3 \mathrm{kpc}$, as compared to $\lesssim 0.4 \mathrm{kpc}$ for 

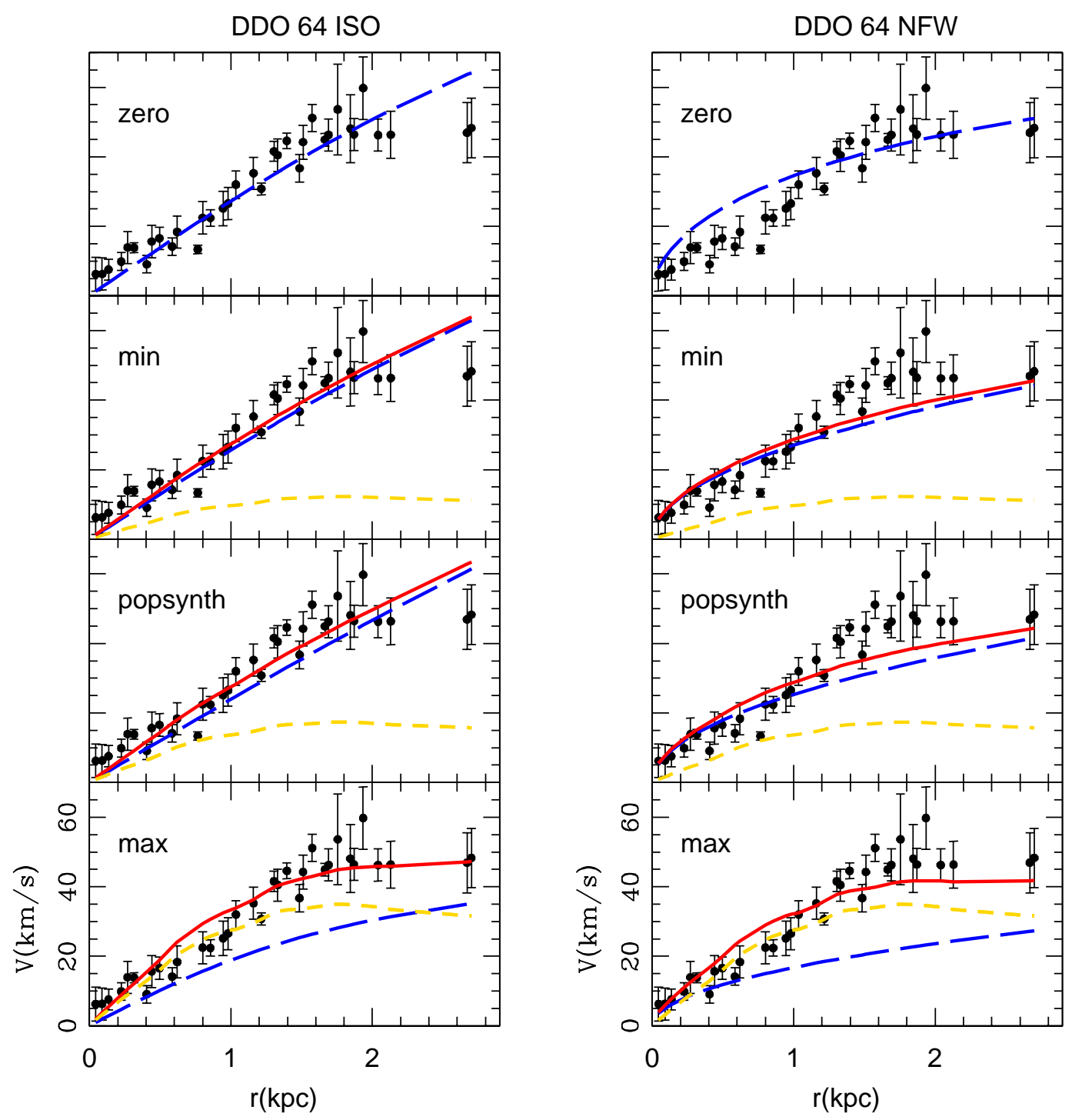

FIG. 8.- Isothermal and NFW halo fits for DDO 64. Line types are described in Figure 5. [See the electronic edition of the Journal for a color version of this figure.]

$\Upsilon_{* P O P}=1.14$. Because the baryons are able to explain so much of the data in the maximum disk case, there is little room left for a dark matter isothermal halo. When this happens, the cored halo becomes very nearly hollow with $\rho_{0}$ decreasing to a very small value and $R_{c}$ increasing to a large number. For the maximum disk case of UGC 4325, we forced an isothermal halo fit by fixing $V_{h}$ to approximately the observed velocities of the outer rotation curve and then varying $R_{c}$ such that $V_{T O T}$ follows the data. In this fit, the resulting $R_{c}$ is a lower limit and $\rho_{0}$ is an upper limit.

F563-V2-As discussed in $\mathrm{K} 06$ for the zero disk case, F563-V2 has too few data points to really distinguish between halo types. This remains true for the other $\Upsilon_{*}$ scenarios as well. The value of $\Upsilon_{*}$ can be turned up to 4.0 in the maximum disk case, leaving essentially no room for an NFW halo; a fit was forced with $c=1.0$. NFW halos could be fit to the zero, minimal, and popsynth cases, however, and the values of the best-fitting concentrations, $c=7.7,8.4$, and 7.8, respectively, are comparable to values expected from simulations. Because of the $c-V_{200}$ degeneracy that allows halos of different $\left(c, V_{200}\right)$ to look the same over a finite range of radius (de Blok et al. 2001), these three NFW fits are essentially indistinguishable. This is an example of where the zero disk assumption is reasonable for LSB galaxies.

F563-1 - The isothermal halo fits the F563-1 data better than the NFW halo, though the values of the bestfitting concentrations are consistent with values expected in $\Lambda$ CDM. The baryons can account for the majority of the observed rotation in the maximum disk case and a forced NFW fit was made with the concentration fixed at 1.0. Stellar population models set $\Upsilon_{* P O P}=1.36$ for F563-1. With this value of the $\Upsilon_{*}$, the stellar rotation curve just grazes the innermost observed rotation curve point. The $\Upsilon_{* M A X}$ can be substantially turned up such 

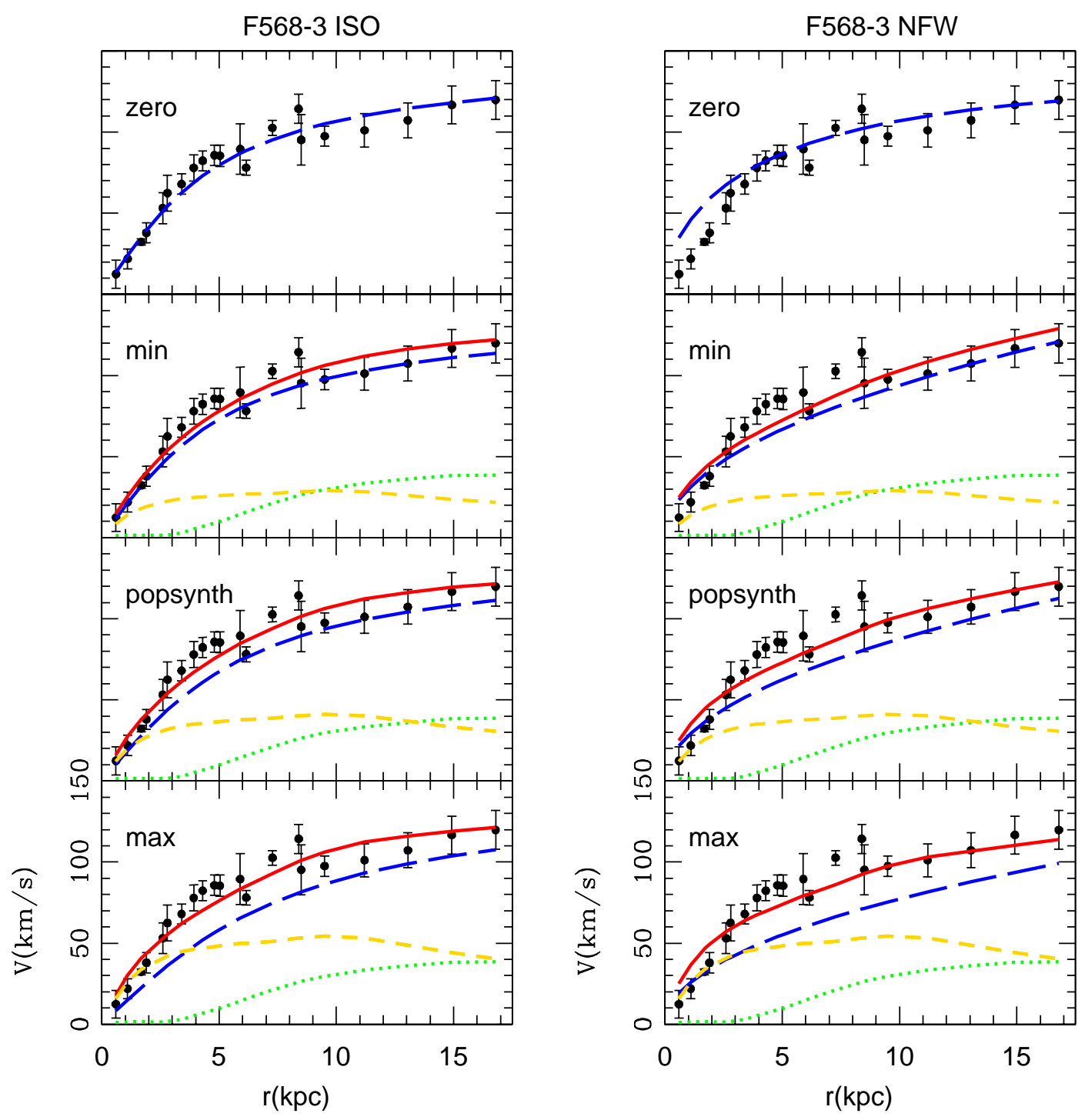

FIG. 9.- Isothermal and NFW halo fits for F568-3. Line types are described in Figure 5. [See the electronic edition of the Journal for a color version of this figure.]

that the stellar rotation curve matches the data more closely. If $\Upsilon_{* M A X}=6.5, V_{*}$ overshoots the innermost rotation curve point, but matches the cluster of points at $\sim 1.5 \mathrm{kpc}$ and even crosses the lower errorbars on points between $5 \mathrm{kpc}$ and $10 \mathrm{kpc}$. The shape of the observed rotation curve is well-matched when $\Upsilon_{* M A X}=$ $10.0 ; V_{*}$ goes through the upper errorbars on points $\lesssim 2$ $\mathrm{kpc}$ and agrees well with the data between $5 \mathrm{kpc}$ and 10 kpc. Fitting the inner observed rotation curve points or the overall rotation curve shape are both equally plausible approaches to defining $\Upsilon_{* M A X}$ (Palunas \& Williams 2000), so we use both values in our halo fits.

F563-1 is a good example of how the choice of $\Upsilon_{*}$ affects the mass models, and the degeneracy between the luminous and dark components. While $\Upsilon_{* P O P}$ is our best estimate for the stars, the data will clearly allow a higher $\Upsilon_{*}$. To constrain the degeneracy between the stars and dark matter in cases like this, we need more information. The stellar velocity dispersion perpendicular to the disk, for instance, would help to put limits on the disk mass (Verheijen et al. 2007).

DDO 64 - As Hi surface density profiles were unavailable for this galaxy, the baryons in the mass models are represented by the stars only. Multicolor photometry was also unavailable, so a $(B-R)=0.9$ color was assumed for this dwarf galaxy (de Blok et al. 1995). DDO 64 is better described by the isothermal halo than the NFW halo; forced $c=1 \mathrm{NFW}$ fits are made for each value of $\Upsilon_{*}$. The stellar rotation curve falls below the observed rotation curve with the exception of two low points in the popsynth case, $\Upsilon_{* P O P}=1.24$. In the maximum disk case with $\Upsilon_{* M A X}=5.0$, however, $V_{*}$ is able to follow the data very well out to just past $1 \mathrm{kpc}$, and is even consistent with a data point at $\sim 1.5 \mathrm{kpc}$. With $\Upsilon_{* M A X}=5.0$, the stars are able to account for most of the observed rotation, and the displayed isothermal halo 

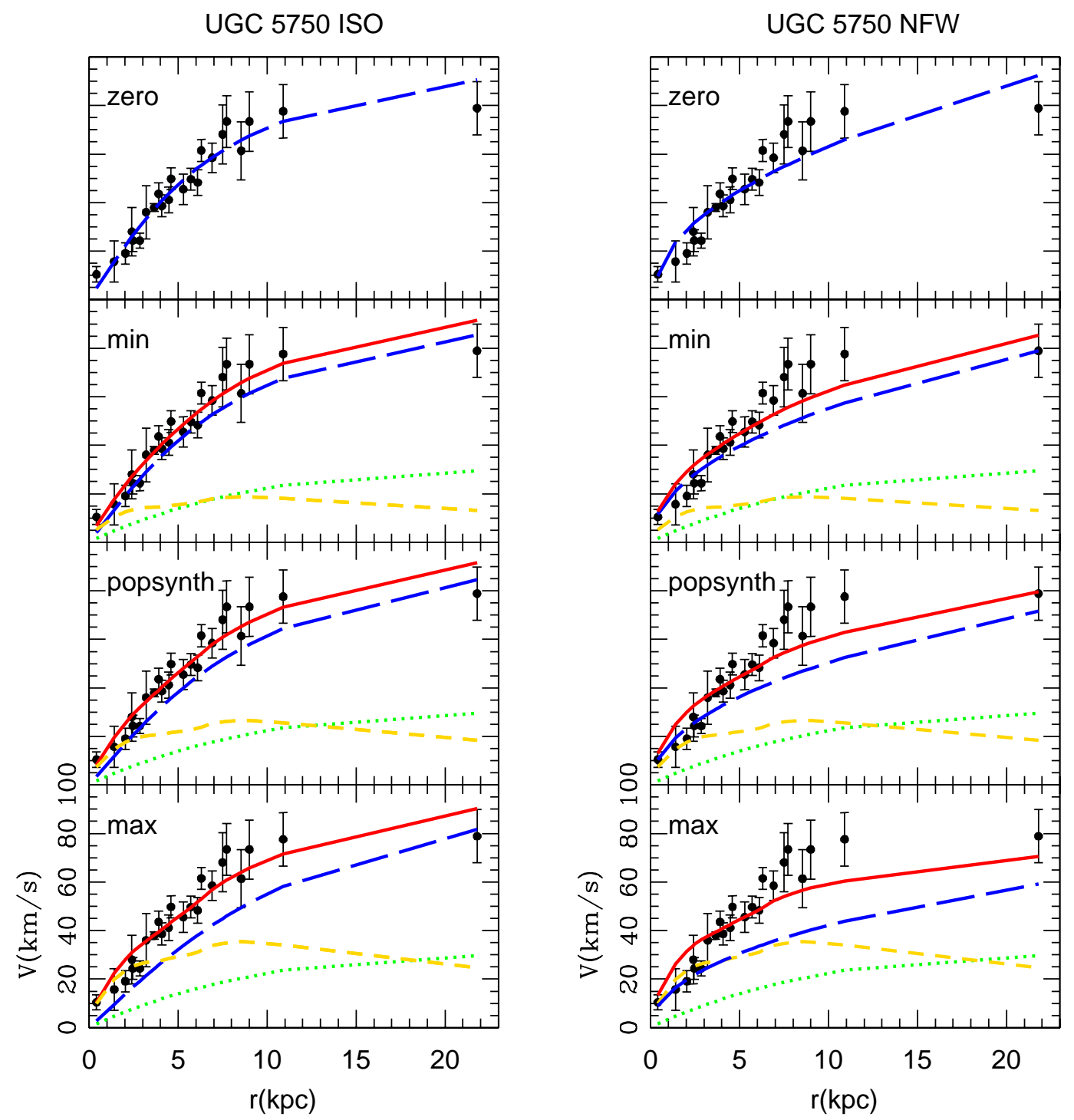

FIG. 10.- Isothermal and NFW halo fits for UGC 5750. Line types are described in Figure 5. [See the electronic edition of the Journal for a color version of this figure.]

fit is the upper limit on $\rho_{0}$ and the lower limit on $R_{c}$.

$\boldsymbol{F 5 6 8 - 3}$ - F568-3 is fit well by isothermal halos; only forced NFW fits could be made to the data. The shape of the stellar rotation curve is not well-matched to the observed rotation curve and is only able to describe the inner $2 \mathrm{kpc}$ of data, even in the maximum disk case.

UGC 5750 - Because multicolor photometry was unavailable, a $(B-R)=0.9$ color was assumed for this galaxy (de Blok et al. 1995). Excellent isothermal fits were made for UGC 5750; the NFW halo provides very poor fits. Like F568-3, there is not a substantial difference between $\Upsilon_{* P O P}$ and $\Upsilon_{* M A X}$.

NGC 4395-Multicolor photometry was unavailable for this galaxy and $\Upsilon_{* P O P}=1.4$ (de Blok \& Bosma 2002) was assumed for the popsynth model. The NFW halo is a slightly better fit to NGC 4395 than the isothermal halo, and the best-fitting concentrations are consistent with expectations from $\Lambda$ CDM. There is a substan- tial difference between $\Upsilon_{* P O P}$ and $\Upsilon_{* M A X}$. For $\Upsilon_{* P O P}$ $=1.4, V_{*}$ is well below the observed rotation curve; however, for $\Upsilon_{* M A X}=9.0, V_{*}$ is able to trace the data out to $8 \mathrm{kpc}$, the entire length of the rotation curve. Because the baryons can explain the observed rotation so well in the maximum disk case, the data want an isothermal halo with an almost hollow core. We force a fit with an upper limit on $\rho_{0}$ and a lower limit on $R_{c}$. The baryons do such a good job of explaining the observed rotation in the maximum disk case that there is not even room for an NFW halo with $c=1.0$. In the NFW maximum disk plot in Figure 11, the magenta line represents the total baryonic rotation curve, $V_{\text {disk }}^{2}=V_{*}^{2}+V_{\text {gas }}^{2}$. It should be noted that the nucleus of NGC 4395 is the least luminous known Seyfert 1 (Filippenko \& Sargent 1989) and may influence the inner rotation curve that we derive.

F583-4 - The isothermal halo is a good description of the data. The NFW halo fits are comparable, although 

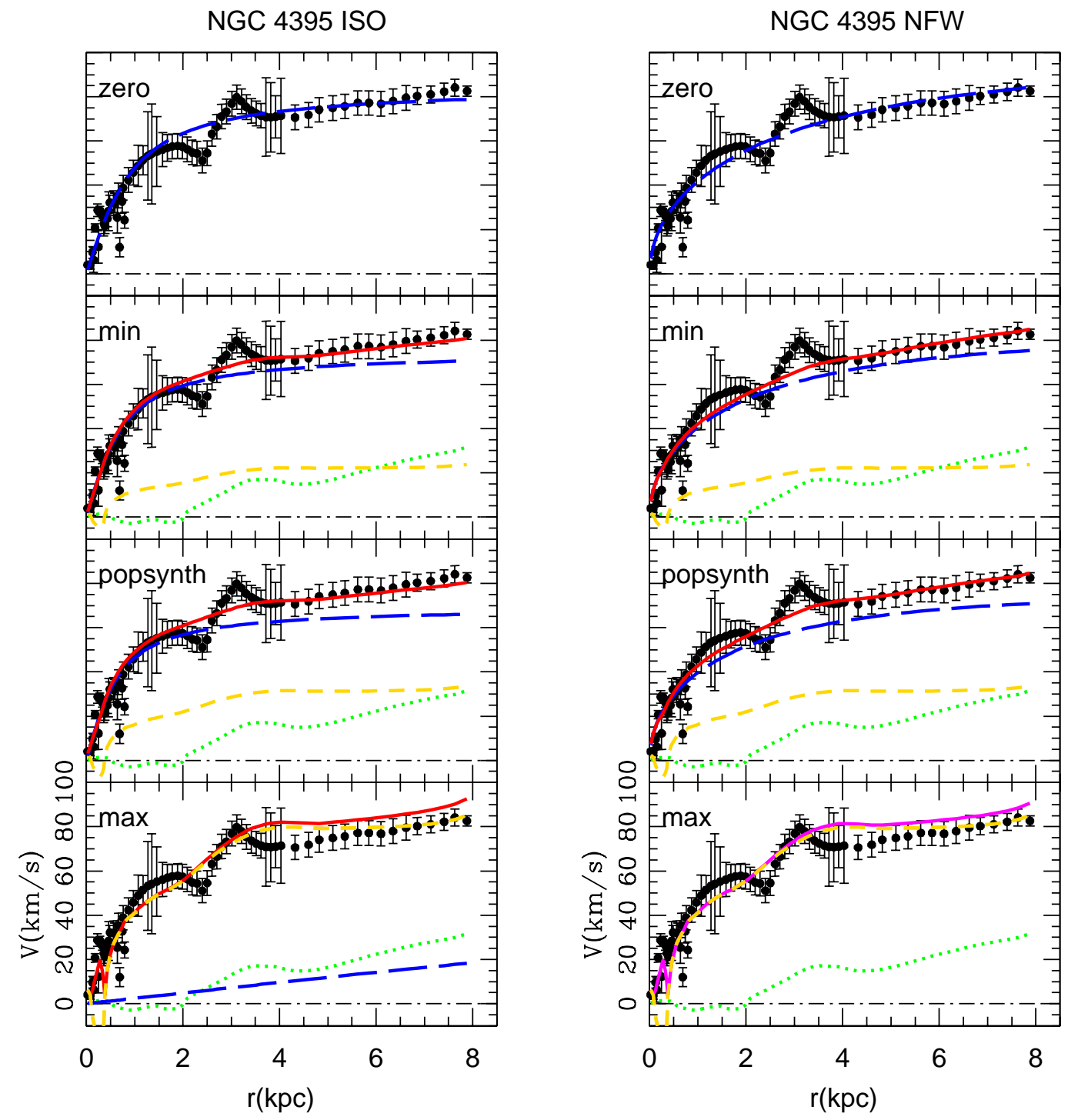

FIG. 11.- Isothermal and NFW halo fits for NGC 4395. Line types are described in Figure 5. The solid magenta line in the maximum disk NFW plot is the total baryonic rotation curve. See text for details. [See the electronic edition of the Journal for a color version of this figure.]

the values of the concentrations are on the low side of expected values from simulations. The stellar rotation curve is far below the data at all radii for the popsynth $\Upsilon_{* P O P}=1.06$. The entire observed rotation curve can be well described by the stellar rotation curve when $\Upsilon_{* M A X}$ is turned up to 10.0. In this case, there is very little room left for a dark matter halo, and only a limiting isothermal halo fit is made. Like NGC 4395, there is not enough velocity left for an NFW halo with $c=1.0$, and the magenta line in the maximum disk NFW plot in Figure 12 represents the total baryonic rotation curve.

F583-1 - F583-1 is better fit by the isothermal halo than the NFW halo. Additionally, the best-fitting NFW concentrations are on the low side of expected values from $\Lambda$ CDM. Like F563-1, we consider two values of $\Upsilon_{* M A X}$. With $\Upsilon_{* M A X}=5.0, V_{*}$ goes through the data within $\sim 1.5 \mathrm{kpc}$. $V_{*}$ goes through the upper errorbars on the inner rotation curve, through the data at $\sim 2 \mathrm{kpc}$, and then through the lower errorbars on the data out to $\sim 3.5 \mathrm{kpc}$ when $\Upsilon_{* M A X}=10.0$.

\section{DISCUSSION}

While one would like to know the true $\Upsilon_{*}$ for each galaxy, our data do not indicate that a particular estimator of $\Upsilon_{*}$ is any better than another. In Figure 14 we show that the parameters of both the isothermal and NFW halo fits do not change much as $\Upsilon_{*}$ changes. This confirms that the details about what is assumed for the stars in LSB galaxies do not really matter. Unfortunately, it also means that without additional information, we cannot constrain $\Upsilon_{*}$ in galaxies like F563-1 and F583-1 where a wide range of $\Upsilon_{*}$ are applicable.

Though the exact assumption about the stars may be unimportant, the stars cannot be entirely ignored. In reality, stars do not have zero mass. In fact, as the velocity contribution from the stars becomes more impor- 
F583-4 ISO

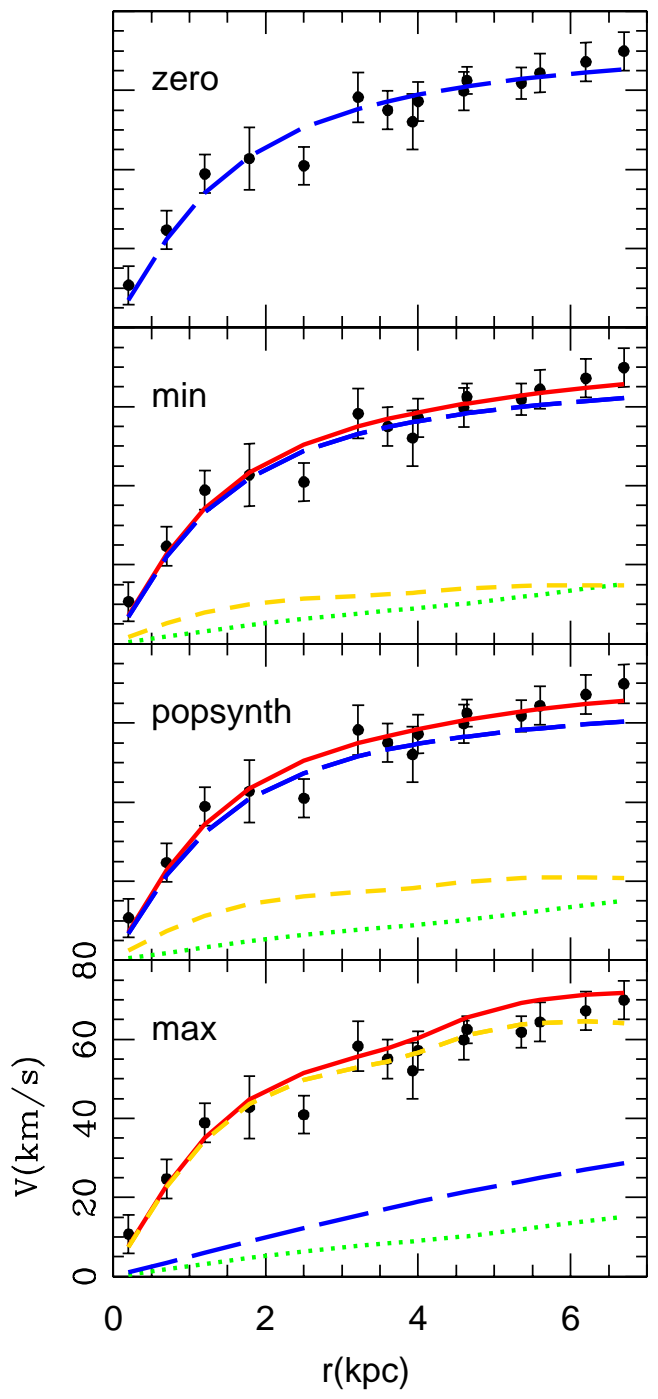

F583-4 NFW

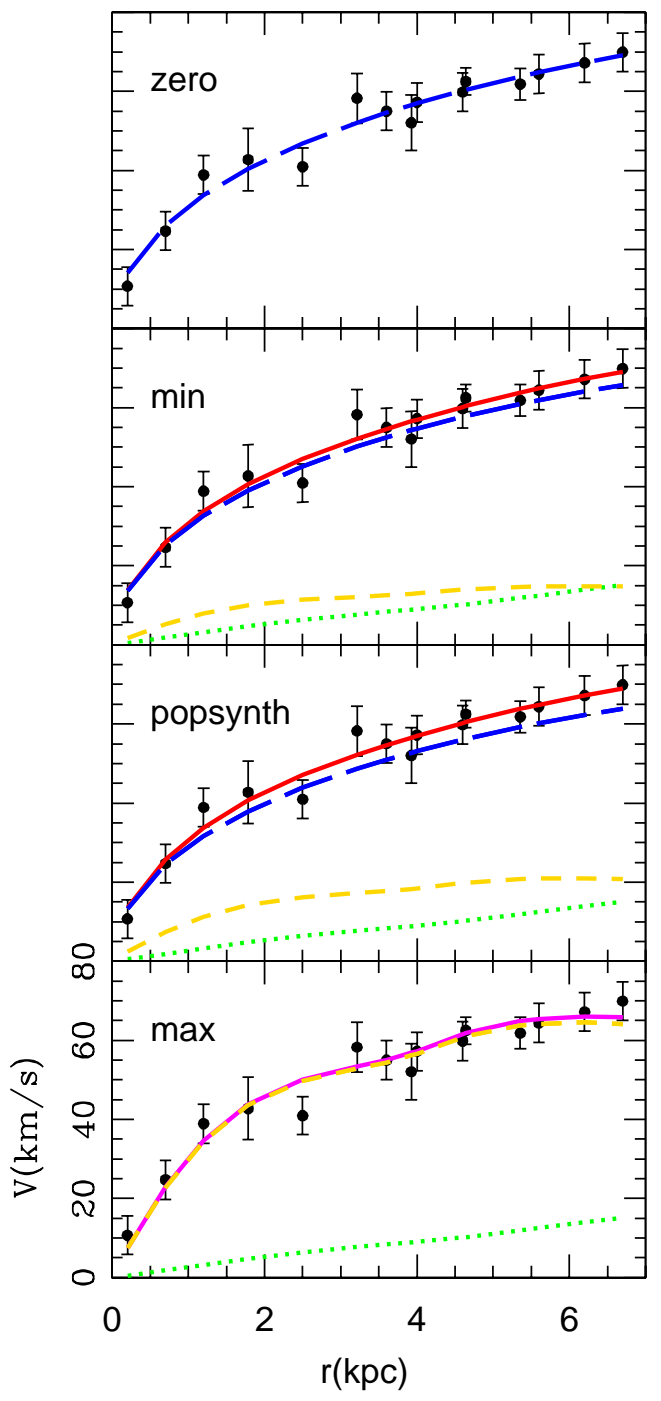

FIG. 12.- Isothermal and NFW halo fits for F583-4. Line types are described in Figure 5. The solid magenta line in the maximum disk NFW plot is the total baryonic rotation curve. See text for details. [See the electronic edition of the Journal for a color version of this figure.]

tant (ie. as $\Upsilon_{*}$ goes up), there is less room for dark matter at the centers of the galaxies. The left panel of Figure 14 illustrates that the isothermal core radius is often larger than $\sim 1 \mathrm{kpc}$. There is also a trend towards larger $R_{c}$ with increasing $\Upsilon_{*}$. This is important to recognize, as it shows that the cusp-core problem is not restricted to the innermost radii only, particularly when stars are allowed to have mass. Also plotted in this figure is the $\rho_{0}-R_{c}$ scaling relation of Kormendy \& Freeman (2004) (their equation 20) and the $\rho_{0}-R_{0}$ relation determined by Spano et al. (2007) (their figure 2). The Kormendy \& Freeman (2004) relation follows the data more closely than the Spano et al. (2007) relation, however the maximum disk case points tend to drift below the Kormendy \& Freeman (2004) relation.

The $c-V_{200}$ plot in the right panel of Figure 14 similarly shows the difficulty of fitting the centrally concentrated NFW halo to the data as the velocity contribution from the baryons becomes larger: as $\Upsilon_{*}$ increases, fewer galaxies can be fitted with an NFW halo. In particular, the velocity contribution from the baryons is significant enough in the maximum disk case that maximum disk and the NFW halo are mutually exclusive.

For comparison, we have also included the predicted $c-V_{200}$ lines for the high $\sigma_{8}$ cosmology of Tegmark et al. (2004, hereafter T04) and the low $\sigma_{8}$ WMAP3 cosmology (Spergel et al. 2007). The width of the bands is $\pm 1 \sigma$, assuming a scatter of $\sigma_{c}=0.14$ (Bullock et al. 2001). The data appear to follow a steeper $c$ - $V_{200}$ relation than both predictions (see also McGaugh et al. 2007), and the concentrations of the DensePak galaxies with NFW fits primarily cluster between the WMAP3 lines. The median concentration of all the zero disk DensePak galaxies is $c=4.5$. At the corresponding $V_{200}$, the median $c$ expected from $\mathrm{T} 04$ is $\sim 8.2$, and the median $c$ expected from WMAP3 is $\sim 6.1$. An alternative method 

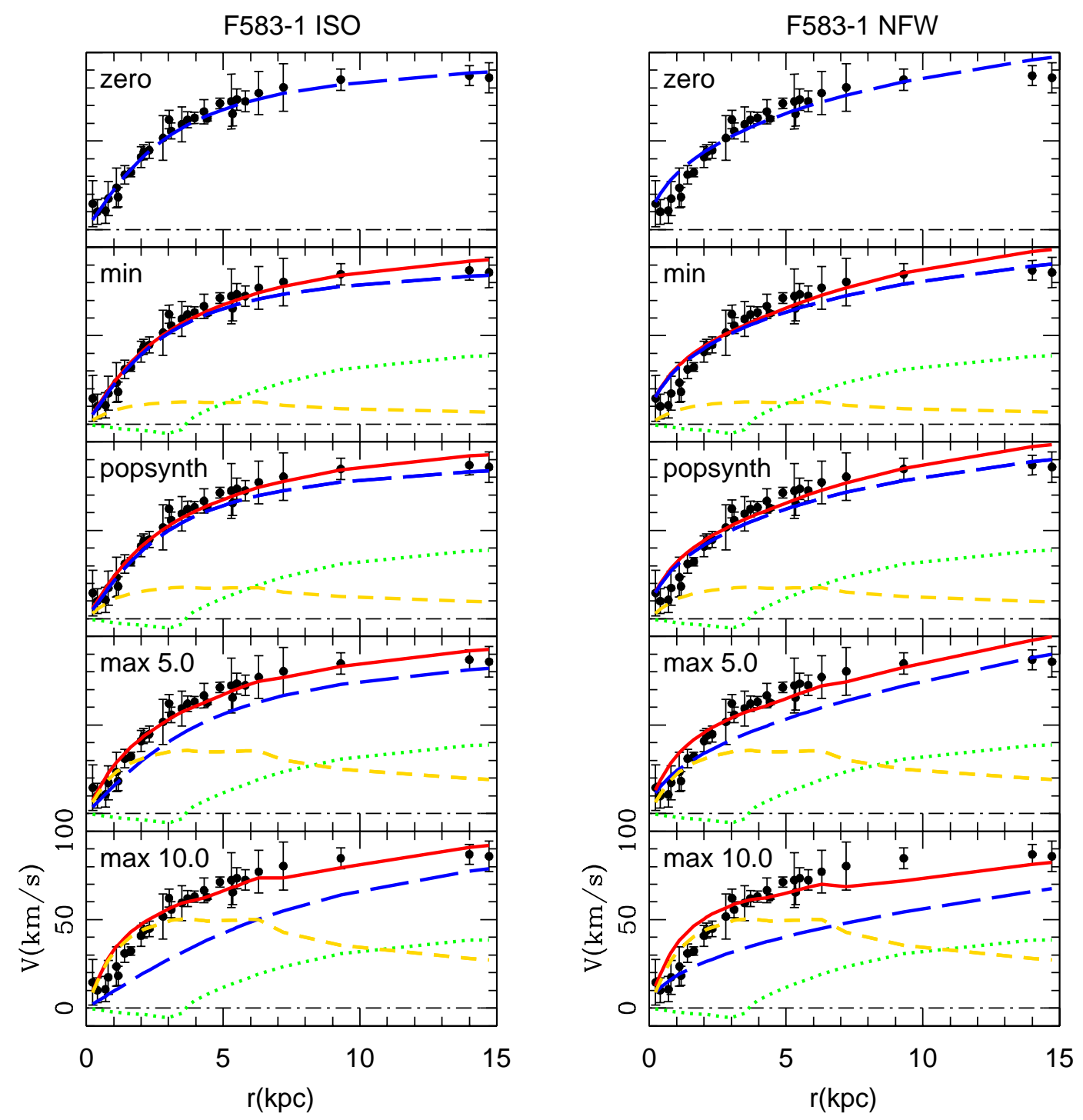

FIG. 13.- Isothermal and NFW halo fits for F583-1. Line types are described in Figure 5. [See the electronic edition of the Journal for a color version of this figure.]

of measuring the halo central density is the $\Delta_{V / 2}$ approach proposed by Alam, Bullock, \& Weinberg (2002). The median $\Delta_{V / 2}$ of all the zero disk DensePak galaxies is $\sim 2.9 \times 10^{5}$. At the corresponding $V_{\max }$, the median $\Delta_{V / 2}$ expected from T04 is $\sim 4.5 \times 10^{5}$ and is $\sim 1.8 \times 10^{5}$ from WMAP3. The low concentrations/central densities observed in the data are more consistent with a power spectrum having a lower amplitude on small (galaxy) scales (see also McGaugh et al. 2003).

\subsection{Cusp Mass Excess}

In $\S 5.2$ we defined a constrained NFW halo, $N F W_{\text {constr }}$, which was constructed to have a cosmologically-consistent concentration by forcing the halo to match the velocities at the outer radii of each galaxy. We can now find $N F W_{\text {constr }}$ halos for the dark matter rotation curves for each determination of $\Upsilon_{*}$. The rotation curves of the $N F W_{\text {constr }}$ halos over-predict the observed velocities interior to where they are forced to agree. We can evaluate the difference between this expected CDM rotation curve and the observed dark matter rotation curve in terms of velocity difference, or alternatively, as a cusp mass excess. We can ask what the difference is between the expected cuspy NFW halo mass and the dark matter mass that is allowed by the data. In the left panel of Figure 15, we show the observed dark matter rotation curves of F568-3 for the zero, minimal, popsynth, and maximum disk models. We also show the constrained NFW halo for each dark matter rotation curve. In the right panel of Figure 15, we plot the same data in terms of mass using $M=V^{2} R / G$. To prevent a cluttered plot, we show only the results for the popsynth model. The solid line is the enclosed mass as a function of radius for the $N F W_{\text {constr }}$ halo. The dashed line is the enclosed mass of the observed dark matter. We have not smoothed the 

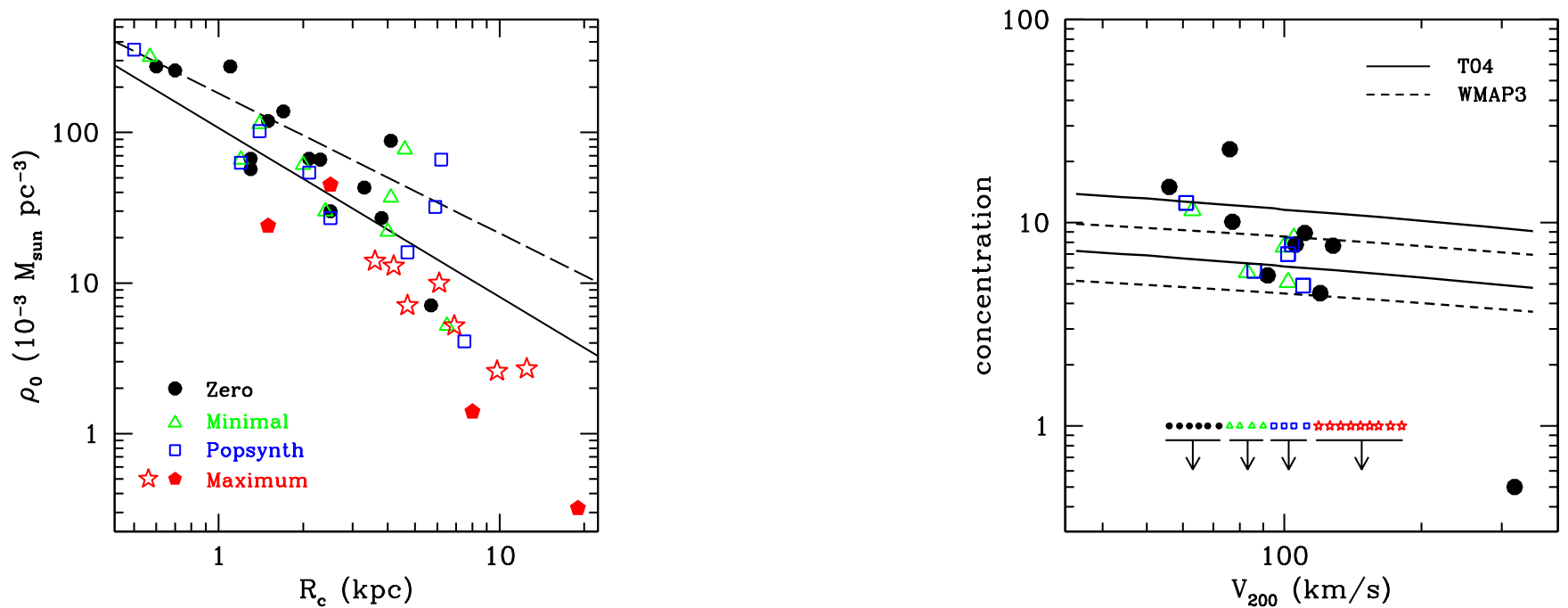

FIG. 14.- Left: Isothermal halo parameters for different assumptions about the baryons. The red pentagons are maximum disk fits where $R_{c}$ is a lower limit and $\rho_{0}$ is an upper limit. The data for NGC 959 are beyond the range of this plot and are not shown. The core radius is often $>1 \mathrm{kpc}$. There is a trend towards larger $R_{c}$ with increasing $\Upsilon_{*}$. For comparison, the solid line is the $\rho_{0}-R_{c}$ scaling law of Kormendy \& Freeman (2004) and the dashed line is the $\rho_{0}-R_{0}$ relation of [Spano et al. (2007). Right: $N F W_{\text {free }}$ halo parameters for different assumptions about the baryons. No NFW fits could be made in the maximum disk case. The solid lines show the range of $c$ - $V_{200}$ predicted by the cosmology of Tegmark et al. (2004), whereas the dashed lines are the predictions for WMAP3 (Spergel et al. 2007). For both cosmologies, the width of the bands is $\pm 1 \sigma$, assuming a scatter of $\sigma_{c}=0.14$ (Bullock et al. 2001). The data show a steeper $c$ - $V_{200}$ relation, and favor the lower concentrations/lower $\sigma_{8}$ predicted by WMAP3. [See the electronic edition of the Journal for a color version of this figure.]
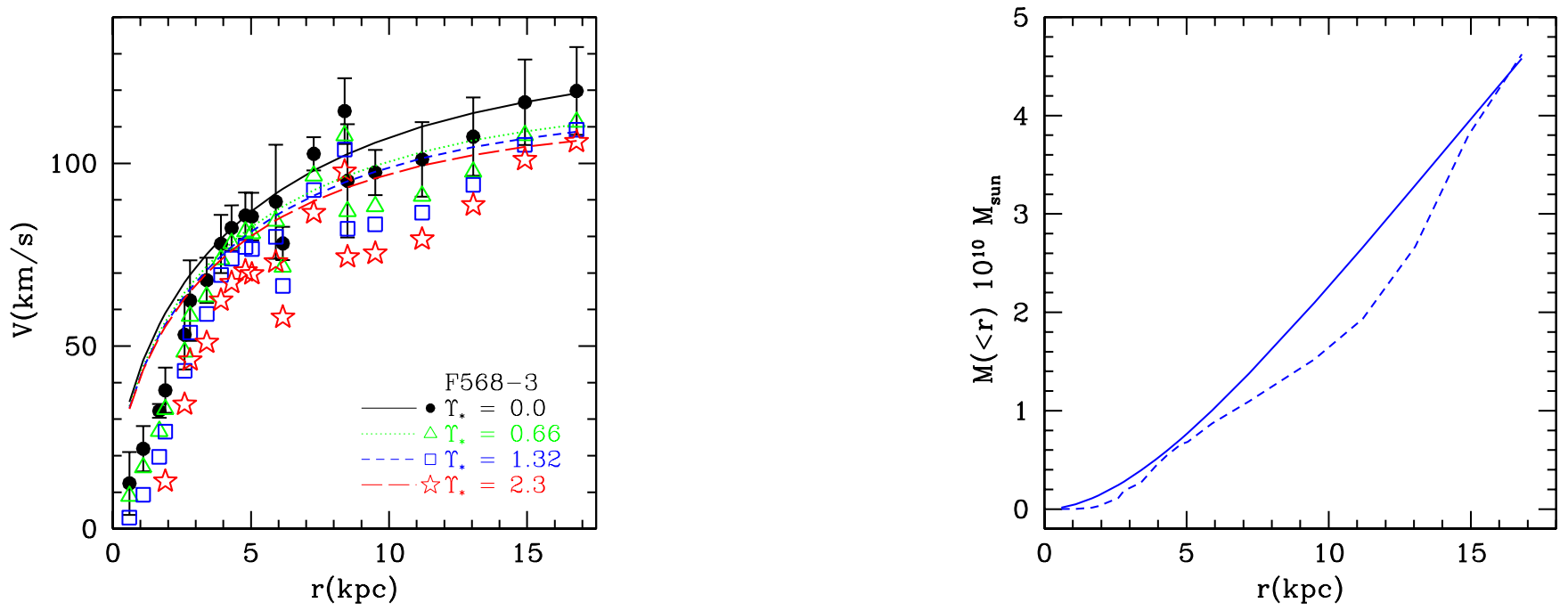

FIG. 15. - Left: Comparison of the dark matter rotation curve for different values of $\Upsilon_{*}$ with the NFW rotation curves expected from cosmology for F568-3. Right: Total dark matter mass as a function of radius for $\Upsilon_{* P O P}$. The solid line is the dark matter mass predicted by CDM; the dashed line is the dark matter mass allowed by the data. The two curves have been forced to meet at large radii; interior to this, CDM predicts more mass at all radii than is actually observed. [See the electronic edition of the Journal for a color version of this figure.]

data, so $V_{D M}$ is a bit jittery between $\sim 6-8 \mathrm{kpc} ; M(r)$, however, is displayed using a smoother version of the data. The $N F W_{\text {constr }}$ halo has been forced to match the data at large radii; interior to this, however, there is less mass observed at all radii than is expected.

In Figure 16 we plot the ratio of the observed dark matter mass to the constrained cusp mass as a function of radius for each galaxy. This ratio approaches 1 at large radii where the data have been forced to agree.
Near the centers of the galaxies there is a substantial cusp mass excess; there is at least two times more mass expected in the cuspy halo than is allowed by the data. At all radii, the cusp mass excess becomes larger as the baryons become increasingly more important.

\subsection{Reconciling the Cusp Mass Excess with Noncircular Motions}



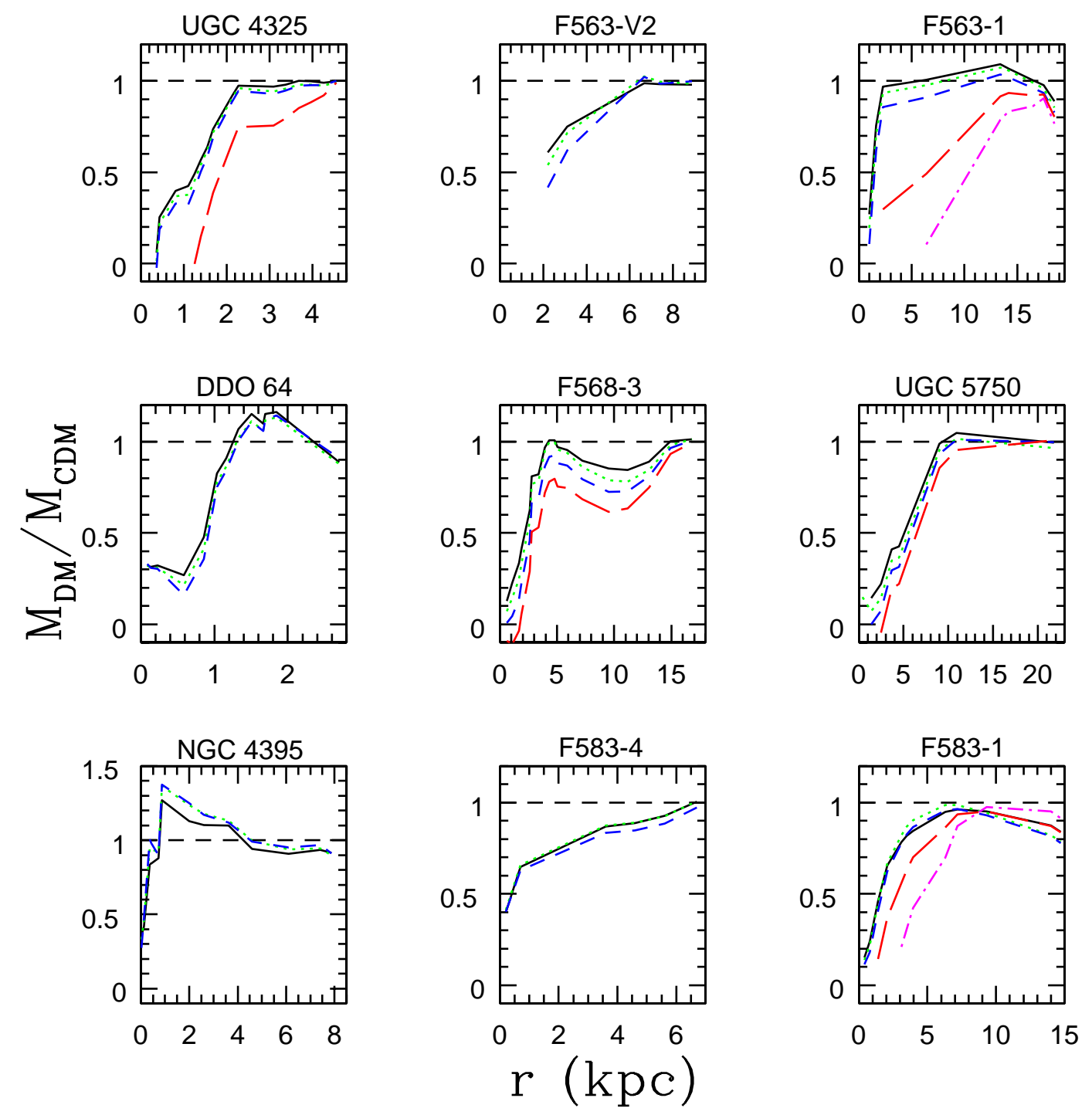

FIG. 16. - Ratio of the observed dark matter mass to the constrained cusp mass as a function of radius. The black (solid), green (dotted), blue (short dash), and red/magenta (long dash/dot-long dash) lines are the zero, minimal, popsynth, and maximum disk cases, respectively. Near the centers of the galaxies there is a substantial cusp mass excess with at least two times more mass expected in the cuspy halo than is allowed by the data. [See the electronic edition of the Journal for a color version of this figure.]

In our analysis so far, we have assumed that the observed velocity is the circular velocity $\left(V_{\text {circ }}=V_{\text {obsv }}\right)$. If noncircular motions are present, the true circular velocity may be underestimated. This is the argument commonly given to explain the discrepancy between the NFW halo and the observations at small radii (e.g. Swaters et al. 2003a; van den Bosch \& Swaters 2001). To determine the true circular velocity, the noncircular motions are added in quadrature to the observed velocities, $V_{\text {circ }}^{2}=$ $V_{\text {rot }}^{2}+\beta \sigma^{2}$, usually assuming an isotropic dispersion $(\beta$ $=3$.

We can invert Figure 16 and determine how large the noncircular motions must be in order to bring the observed dark matter velocity into agreement with the $N F W_{\text {constr }}$ velocity. In Figure 17 we plot the required $\sigma(r)$, assuming an isotropic dispersion, for each galaxy.
In general, most of the galaxies need about $20 \mathrm{~km} \mathrm{~s}^{-1}$ noncircular motions at inner radii for the data to be consistent with the expectations of CDM. This is roughly twice as large as the velocity dispersion in the DensePak data (K06). It is also important to recognize the effect of stars not having zero mass. Instead of just having to convince ourselves that $\sim 20 \mathrm{~km} \mathrm{~s}^{-1}$ noncircular motions should be added to the data, we actually must be willing to consider noncircular motions an additional $5-10 \mathrm{~km}$ $\mathrm{s}^{-1}$ higher when $\Upsilon_{*} \neq 0$. Because we have forced the halo velocity to match the observed velocity at large radius, our derived $\sigma(r)$ often show a steep drop to $0 \mathrm{~km}$ $\mathrm{s}^{-1}$ from $\sim 20 \mathrm{~km} \mathrm{~s}^{-1}$. Strictly speaking, this behavior is unphysical, and a more continuous model could be derived. If $\sigma$ contributes at large radii, however, that implies that the halo has even more dark matter and $\sigma$ at small radii would have to be even higher. In a forth- 

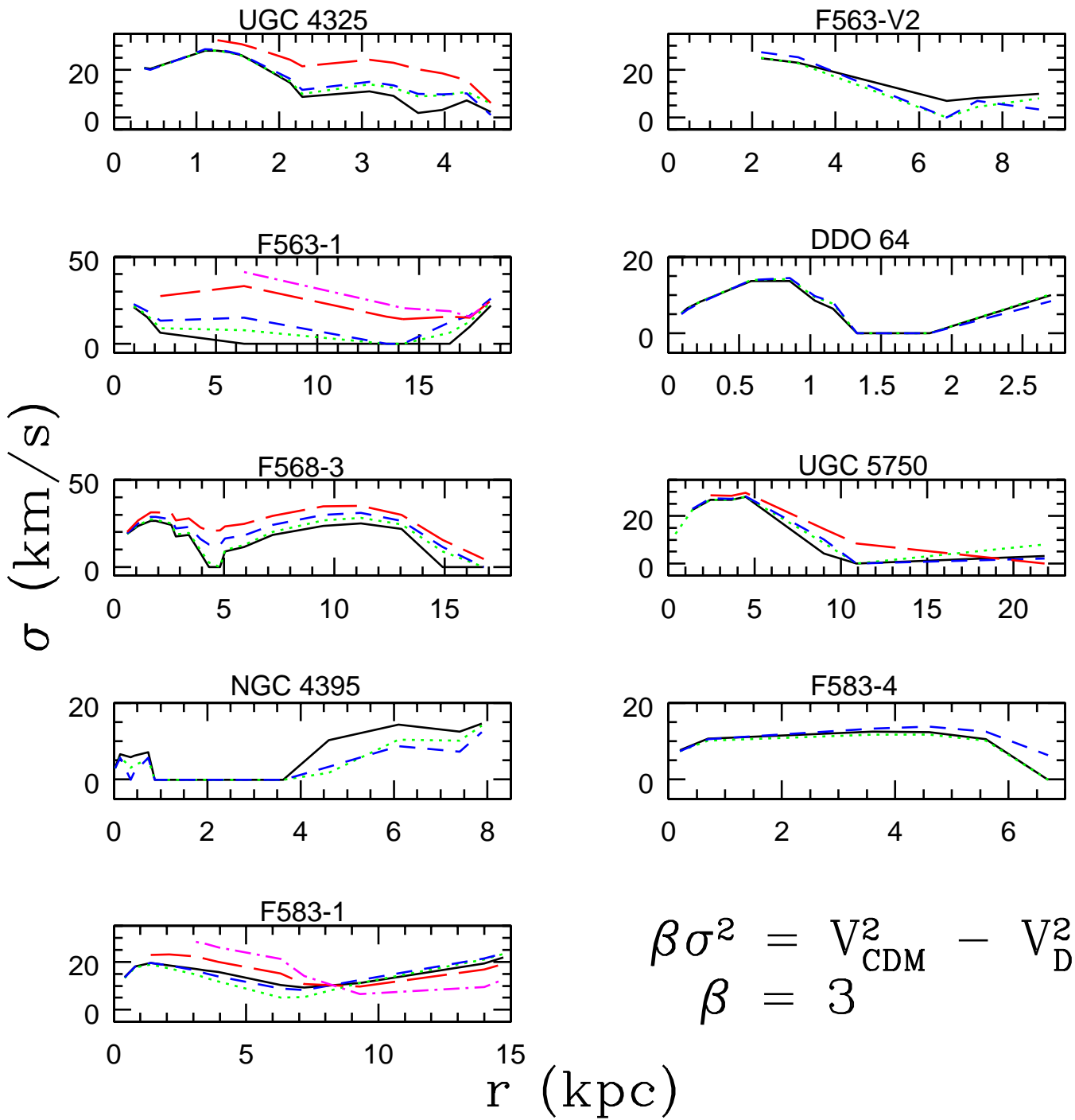

FIG. 17.- The required noncircular motions, assuming an isotropic dispersion, to reconcile the difference between the $N F W_{\text {constr }}$ velocity and the observed dark matter velocity. The black (solid), green (dotted), blue (short dash), and red/magenta (long dash/dot-long dash) lines are the zero, minimal, popsynth, and maximum disk cases, respectively. [See the electronic edition of the Journal for a color version of this figure.]

coming paper, we model the NFW halo and noncircular motions, and Figure 17 provides us with testable predictions. For instance, we can use the results to test whether the observations are consistent with $N F W+\sigma(r)$.

\section{CONCLUSIONS}

We have presented updated DensePak velocity fields, rotation curves and zero disk halo fits for three galaxies from K06. We have also presented the velocity fields, derived rotation curves and zero disk halo fits for six galaxies previously unobserved with DensePak. Overall, we find the isothermal halo to be a better description of both the shape of the rotation curves and the central densities of these nine galaxies in the limit of zero disk than the NFW halo. When NFW fits could be made, the concentrations were often beyond the range of values expected for $\Lambda$ CDM. We also find that the quality of both the isothermal and NFW halo fits is greatly improved when the radial range of the data extends into the flat part of the rotation curve.

For those DensePak galaxies with photometry, we have also presented isothermal and NFW halo fits for four assumptions of the stellar mass-to-light ratio, $\Upsilon_{*}$. We have tested the zero, minimal, popsynth, and maximum disk cases. We have found that the NFW halo is a poor description of the data, and that the NFW halo and maximum disk are mutually exclusive. There is a substantial cusp mass excess near the centers of the galaxies with at least two times more mass expected in the cuspy CDM halo than is allowed by the data. Most galaxies in the sample require $\sim 20 \mathrm{~km} \mathrm{~s}^{-1}$ noncircular motions to reconcile the differences between observations and the NFW halo. Even larger noncircular motions are required when stars are allowed to have mass. We have also found the 
data to favor a low $\sigma_{8}$ cosmology.

\section{ACKNOWLEDGEMENTS}

We thank James Bullock for providing the theoretical predictions for $\Delta_{V / 2}$. The work of R. K. D. and S. S. M. was supported by NSF grant AST0505956. R. K. D. is also supported by an NSF Astronomy \& Astrophysics Postdoctoral Fellowship under award AST0702496. This paper was part of R. K. D. 's Ph. D. dissertation at the University of Maryland. The work of W. J. G. d. B. is supported by the South African Research Chairs Initia- tive of the Department of Science and Technology and National Research Foundation. This research has made use of the NASA/IPAC Extragalactic Database (NED) which is operated by the Jet Propulsion Laboratory, California Institute of Technology, under contract with the National Aeronautics and Space Administration. Our velocity field plots were made using a modified version of the program found at http://www. astro.wisc.edu/\$\sim\$mab/research/densepak/ DP/dpidl.html

\section{REFERENCES}

Alam, S. M. K., Bullock, J. S., \& Weinberg, D. H. 2002, ApJ, 572,34

Avila-Reese, V., Firmani, C., \& Hernandez, X. 1998, ApJ, 505, 37

Baggett, W. E., Baggett, S. M., \& Anderson, K. S. J. 1998, AJ, 116,1626

Begeman, K. G. 1989, A\&A, 223, 47

Bell, E. F., McIntosh, D. H., Katz, N., \& Weinberg, M. D. 2003, ApJS, 149, 289

Borriello, A., \& Salucci, P. 2001, MNRAS, 323, 285

Bullock, J. S., Kolatt, T. S., Sigad, Y., Somerville, R. S., Kravtsov, A. V., Klypin, A. A., Primack, J. R., \& Dekel, A. 2001, MNRAS, 321, 559

Chemin, L., et al. 2004, IAUS, 220, 333

Cole, S., \& Lacey, C. 1996, MNRAS, 281, 716

de Blok, W. J. G., \& Bosma, A. 2002, A\&A, 385, 816

de Blok, W. J. G., Bosma, A., \& McGaugh, S. S. 2003, MNRAS, 340,657

de Blok, W. J. G., \& McGaugh, S. S. 1996, ApJ, 469, L89

- . 1997, MNRAS, 290, 533

de Blok, W. J. G., McGaugh, S. S., \& Rubin, V. C. 2001, AJ, 122,2396

de Blok, W. J. G., McGaugh, S. S., \& van der Hulst, J. M. 1996, MNRAS, 283, 18

de Blok, W. J. G., \& van der Hulst, J. M. 1998, A\&A, 336, 49

de Blok, W. J. G., van der Hulst, J. M., \& Bothun, G. D. 1995, MNRAS, 274, 235

de Jong, R. S. 1996, A\&AS, 118, 557

Diemand, J., Zemp, M., Moore, B., Stadel, J., \& Carollo, M. 2005, MNRAS, 364, 665

Filippenko, A. V., \& Sargent, W. L. W. 1989, ApJ, 342, L11

Flores, R. A., \& Primack, J. R. 1994, ApJ, 427, L1

Fuchs, B. 2003, Ap\&SS, 284, 719

Gentile, G., Burkert, A., Salucci, P., Klein, U., \& Walter, F. 2005 ApJ, 634, L145

Gentile, G., Salucci, P., Klein, U., \& Granato, G. L. 2007, MNRAS, 375, 199

Gentile, G., Salucci, P., Klein, U., Vergani, D., \& Kalberla, P. 2004, MNRAS, 351, 903

Heraudeau, P., \& Simien, F. 1996, A\&AS, 118, 111

James, P. A., et al. 2004, A\&A, 414, 23

Klypin, A., Kravtsov, A. V., Bullock, J. S., \& Primack, J. R. 2001, ApJ, 554, 903

Kormendy, J., \& Freeman, K. C. 2004, IAUS, 220, 377

Kuzio de Naray, R., McGaugh, S. S., de Blok, W. J. G., \& Bosma, A. 2006, ApJS, 165, 461 (K06)

Marchesini, D., D'Onghia, E., Chincarini, G., Firmani, C., Conconi, P., Molinari, E., \& Zacchei, A. 2002, ApJ, 575, 801

McGaugh, S. S. 2005, ApJ, 632, 859

McGaugh, S. S., Barker, M. K., \& de Blok, W. J. G. 2003, ApJ, 584,566

McGaugh, S. S., \& de Blok, W. J. G. 1998, ApJ, 499, 41

McGaugh, S. S., de Blok, W. J. G., Schombert, J. M., Kuzio de Naray, R., \& Kim, J. H. 2007, ApJ, 659, 149

McGaugh, S. S., Rubin, V. C., \& de Blok, W. J. G. 2001, AJ, 122,2381
Mihos, J. C., Spaans, M., \& McGaugh, S. S. 1999, ApJ, 515, 89 Moore, B., Quinn, T., Governato, F., Stadel, J., Lake, G. 1999, MNRAS, 310, 1147

Navarro, J. F., Frenk, C. S., \& White, S. D. M. 1996, ApJ, 462, 563

- . 1997, ApJ, 490, 493

Navarro, J.F., et al. 2004, MNRAS, 349, 1039

Osterbrock, D. E., Fulbright, J. P., Martel, A. R., Keane, M. J., Trager, S. C., \& Basri, G. 1996, PASP, 108, 277

Palunas, P., \& Williams, T. B. 2000, AJ, 120, 2884

Reed, D., Gardner, J., Quinn, T., Stadel, J., Fardal, M., Lake, G., \& Governato, F. 2003, MNRAS, 346, 565

Regan, M. W., Thornley, M. D., Helfer, T. T., Sheth, K., Wong, T., Vogel, S. N., Blitz, L., \& Bock, D. C. J. 2001, ApJ, 561218

Rhee, G., Valenzuela, O., Klypin, A., Holtzman, J., \& Moorthy, B. 2004, ApJ, 617, 1059

Rosenberg, J. L., \& Schneider S. E. 2003, ApJ, 585, 256

Schombert, J. M., Bothun, G. D., Impey, C. D., \& Mundy, L. G. 1990, AJ, 100, 1523

Simon, J. D., Bolatto, A. D., Leroy, A., \& Blitz, L. 2003, ApJ, 596,957

Simon, J. D., Bolatto, A. D., Leroy, A., Blitz, L., \& Gates, E. L. 2005, ApJ, 621, 757

Spano, M., Marcelin, M., Amram, P., Carignan, C., Epinat, B., \&

Hernandez, O. 2007, MNRAS, in press (arXiv:0710.1345)

Spergel, D. N., et al. 2007, ApJS, 170, 377

Stil, J. 1999, Ph.D. Thesis, University of Leiden

Swaters, R. A. 1999, Ph.D. Thesis, University of Groningen

Swaters, R. A., \& Balcells, M. 2002, A\&A, 390, 863

Swaters, R. A., Madore, B. F., van den Bosch, F. C., \& Balcells, M. 2003a, ApJ, 583, 732

Swaters, R. A., van Albada, T. S., van der Hulst, J. M., \& Sancisi, R. 2002, A\&A, 390, 829

Swaters, R. A., Verheijen, M. A. W., Bershady, M. A., \& Andersen, D. R. 2003b, ApJ, 587, L19

Tegmark, M., et al. 2004, Phys. Rev. D, 69, 103501

Tully, R. B. 1988, Nearby Galaxies Catalogue (Cambridge: Cambridge Univ. Press)

van den Bosch, F. C., Robertson, B. E., Dalcanton, J. J., \& de Blok, W. J. G. 2000, AJ, 119, 1579

van den Bosch, F. C., \& Swaters, R. A. 2001, MNRAS, 325, 1017

van der Hulst, J. M., Skillman, E. D., Smith, T. R., Bothun,

G. D., McGaugh, S. S., \& de Blok, W. J. G. 1993, AJ, 106, 548

van der Kruit, P. C., \& Searle, L. 1981, A\&A, 95, 105

van Zee, L., \& Haynes, M. P. 2006, ApJ, 636, 214

van Zee, L., Haynes, M. P., Salzer, J. J., \& Broeils, A. H. 1997, AJ, 113,1618

Verheijen, M., \& de Blok, W. J. G. 1999, Ap\&SS, 269, 673

Verheijen, M. A. W., Bershady, M. A., Swaters, R. A., Andersen, D. R., \& Westfall, K. B. 2007, ISLAND UNIVERSES, Astrophysics and Space Science Proceedings. ISBN 978-1-4020-5572-0. Springer, 2007, p. 95 